\title{
Surgery in patients with small cell lung cancer: A period propensity score matching analysis of the Seer database, 2010-2015
}

\author{
XIAOJUN DU ${ }^{1}$, DAN TIAN ${ }^{2}$, LANGBO LIU ${ }^{1}$, ZHONGBEN TANG $^{1}$, JIARONG XIAO $^{1}$, WEN LIU ${ }^{1}$, \\ SHIZHANG YUAN $^{3}, \mathrm{XI} \mathrm{CAO}^{3}$, HAIYU ZHOU $^{2}$ and JIAN ZHANG ${ }^{1}$ \\ ${ }^{1}$ Department of Thoracic Surgery, The Affiliated Hospital of Guizhou Medical University, \\ Guiyang, Guizhou 550004; ${ }^{2}$ Department of Thoracic Surgery, Guangdong General Hospital, \\ Guangdong Academy of Medical Sciences, Guangzhou, Guangdong 510080; ${ }^{3}$ Department of Thoracic Surgery, \\ Guiyang Public Health Clinical Center, Guiyang, Guizhou 550003, P.R. China
}

Received November 26, 2018; Accepted August 7, 2019

DOI: $10.3892 / \mathrm{ol} .2019 .10792$

\begin{abstract}
Surgery as a therapeutic modality for non-small cell lung cancer is widely accepted in clinical practice. However, the role of surgery for small cell lung cancer (SCLC) remains controversial. Therefore, in the present study a period propensity score matching analysis using the Surveillance, Epidemiology and End Results (SEER) Registry database was performed to investigate the role of surgery on survival in patients with SCLC. Patients with SCLC between January 2010 and December 2015 were identified from the SEER database, and individual data for each case regarding general clinical characteristics, surgery of primary site (SPS), cause-specific death classification and survival time were retrieved. Differences of cause-specific survival (CSS) between subgroups were estimated by the log-rank test. Cox regression analysis was used to evaluate the effects of multiple variables on CSS, and differences between the incidences of cause-specific death were examined using a $\chi^{2}$ test. A total of 1,707 records met the inclusion criteria and were retrieved for analysis. There were significant differences of CSS in the clinicopathological features of $\mathrm{N}(\mathrm{P}=0.01)$, Stage $(\mathrm{P}<0.01)$ and Surgery $(\mathrm{P}<0.01)$ when comparing non-surgery with surgery, and in $\mathrm{N}(\mathrm{P}<0.001)$, Stage $(\mathrm{P}=0.006)$ and Surgery $(\mathrm{P}=0.049)$ when comparing sublobectomy with lobectomy or bilobectomy (lobe/s). Patients who did not receive surgery $(\mathrm{P}<0.001)$ or who received sublobectomy $(\mathrm{P}=0.03)$ had an increased risk of mortality when compared with patients who received surgery and lobe/s. The findings of the present study indicate that surgery should be taken into consideration when an initial
\end{abstract}

Correspondence to: Dr Jian Zhang, Department of Thoracic Surgery, The Affiliated Hospital of Guizhou Medical University, 28 Guiyi Street, Yunyan, Guiyang, Guizhou 550004, P.R. China

E-mail: 280827166@qq.com

Key words: small cell lung cancer, surgery, sublobectomy, lobectomy, pneumonectomy, survival treatment strategy is made in patients for patients with SCLC at clinical stage I-IIA (T1-2,N0,M0), regardless of whether they are $>50$ years of age, their sex, histology and grade. The results suggest that certain patients with SCLC with stage IIB (N1) can also benefit from lobe/s, although further investigation is required. In addition, lobe/s is preferable to sublobectomy when surgery is performed. However, the present study was unable to comprehensively analyze the efficacy of pneumonectomy for SCLC.

\section{Introduction}

Small cell lung cancer (SCLC) represents $13-15 \%$ of all lung cancer diagnoses in the United States between 2006 and $2017(1,2)$. Similar to non-small cell lung cancer, the primary risk factor for SCLC remains smoking tobacco (3). Early diagnosis of SCLC is challenging due to the lack of specific symptoms and its extremely aggressive nature which is characterized by rapid tumor growth, quick doubling time and early metastasis. A statistically significant improvement in 2- and 5-year survival in limited- and extensive-stage SCLC cohorts from the Surveillance, Epidemiology, and End Results (SEER) Registry database, analyzed by Joinpoint regression, has been reported (1). However, the prognosis of patients with SCLC is poor with a 5-year survival rate of $<5 \%$ and an average overall survival time of only 2-4 months for patients who do not receive effective treatment (4). At present, platinum and etoposide remain the preferred first-line chemotherapy regimen for the treatment of extensive-stage SCLC, and surgery is recommended for patients with limited-stage SCLC (5). Although SCLC is sensitive to chemotherapy and radiotherapy, the majority of patients relapse or progress after first-line therapy. Surgery in the form of lobectomy is a potential option for TNM stage I (T1-2N0M0) without mediastinal or supraclavicular involvement (6). However, the role of surgery in SCLC treatment remains controversial. Therefore, the present study performed a period propensity score matching analysis using the SEER database to examine the effects of surgery on survival in patients with SCLC. 


\section{Materials and methods}

Patients and methods. This was a retrospective, population-based study using cases registered in the SEER database made publicly available through online access. Data were retrieved using the Surveillance Research Program, National Cancer Institute SEER*Stat software (seer.cancer. gov/seerstat) version 8.3.5. Informed consent from the study population was waived, as the authors had no access to the identities of the patients, and no identifiable patient information was included.

Data collection. The following database was used for selection of cases: SEER Program (www.seer.cancer.gov) SEER*Stat Database: Incidence-SEER 18 Regs Research Data + Hurricane Katrina Impacted Louisiana Cases, Nov 2017 Sub (2000-2015) <Katrina/Rita Population Adjustment> - Linked To County Attributes-Total U.S., 1969-2016 Counties, National Cancer Institute, DCCPS, Surveillance Research Program, released April 2018, based on the November 2017 submission. Only patients with SCLC [based on International Classification of Diseases for Oncology, 3rd edition (ICD-O-3) (7) codes: 8041/3-8045/3] between January 2010 and December 2015 were included in the present study. The exclusion criteria were: i) An ambiguous or unknown classification of observed clinical characteristics, ii) cause of death to site (COD) recode not as 'Alive' or 'Lung and Bronchus', iii) distant metastasis at the brain, liver and lung, iv) M1, v) T0 and finally vi) a survival time of $<1$ month (Fig. 1). Individual data for each case were retrieved from the database including sex, age at diagnosis, race, histology, grade, surgery to primary site (SPS), Tumor-Node-Metastasis (TNM) stage (8), COD and survival time.

Subgroup definitions. In the SEER database, grades were recorded as follows: i) Grade I, well differentiated; ii) grade II, moderately differentiated; iii) grade III, poorly differentiated and iv) undifferentiated and anaplastic. SPS was divided into: i) Non-surgery, no surgery of primary site; ii) Sublobectomy, Partial/Wedge/Segmental Resection, Lingulectomy, Partial Lobectomy, Sleeve Resection iii) lobe/s, lobectomy or bilobectomy; iv) Pneumonectomy. The T, N, M and Stage were recorded in the database accordingly to the AJCC cancer staging manual, 7 th ed. (8).

Statistical analyses. Statistical analysis were performed using Stata 15.0 (Stata Corp. LLC). The propensity score matching was performed using the 'psmatch2' module in the software. Student's t-test was used to analyze the differences of means between two samples. Differences of cause-specific survival (CSS) between subgroups and the role of surgery in each subgroup was estimated using the Kaplan-Meier product method and compared by a log-rank test. Cox regression analysis was used to evaluate the effects of multiple variables on survival. The difference of incidence of COD was examined using $\chi^{2}$ test. Quantitative data were converted into categorical data, with the exception of survival time. All statistical tests were two-sided and $\mathrm{P}<0.05$ was considered to indicate a statistically significant difference.

\section{Results}

Patients selection and demography of included patients. Based on the patient selection criteria described previously (Fig. 1), 221,646 records of lung cancer between January 2010 and December 2015 were identified from the SEER database. Among them, 28,335 (12.78\%) were SCLC, and 1,707 met the inclusion criteria of the present study and were subsequently extracted for analysis. After propensity score matching, 294 pairs were selected for comparison between non-surgery and surgery, 84 pairs were selected for comparison between sublobectomy and lobe/s and 10 pairs were selected for comparison between lobe/s and pneumonectomy. The results of the propensity score matching are presented in Tables I and II, and Fig. 2. Following matching, the clinicopathological features of grade, histology, stage, $\mathrm{T}$ and $\mathrm{N}$ were balanced in the non-surgery vs. surgery group, as well as $\mathrm{T}$ in the sub-lobectomy vs. lobe/s group and $\mathrm{T}$ and stage in the lobe/s vs. pneumonectomy group $(\mathrm{P}>0.05$ in the matched groups; $\mathrm{P}<0.05$ in the unmatched groups) (Table I). The mean and median biases in the matched groups were lower compared with those in the unmatched groups, and the overall differences in the clinicopathological features between the three different surgical groups were statistically insignificant $(\mathrm{P}=0.13$, 0.96 and 0.28 , respectively) (Table II; Fig. 2). Fig. 3 shows the distribution of survival time and age for each surgical group.

Role of surgery in SCLC. The potential prognostic factors were analyzed by univariate analysis using the Kaplan-Meier method and were compared with the log-rank test (Table III), which revealed that there were significant differences in CSS in $\mathrm{N}(\mathrm{P}=0.01)$, Stage $(\mathrm{P}<0.001)$, and Surgery $(\mathrm{P}<0.001)$ when comparing non-surgery with surgery, and in $\mathrm{N}(\mathrm{P}<0.001)$, Stage $(\mathrm{P}=0.006)$, and Surgery $(\mathrm{P}=0.049)$ when comparing sublobectomy with lobe/s (Fig. 4). However, the difference was not significant when comparing lobe/s with pneumonectomy. Cox regression analysis, (Table IV), which included all characteristics for clinical purposes revealed that the differences were significant for age $[\mathrm{P}<0.001$; hazard ratio $(\mathrm{HR})=1.21$, 95\% confidence interval (CI) 1.07-1.36] and surgery $(\mathrm{P}<0.001$, HR $0.59,95 \% \mathrm{CI}, 0.47-0.76$ ) when comparing non-surgery with surgery. However, no significant differences were detected when comparing sublobectomy with lobe/s, and lobe/s with pneumonectomy. The results of the survival functions of the clinicopathological features stratified by surgery are presented in Table V and Fig. 5. No statistical analysis was performed for certain subclinical features since the testing was not possible when no failures were observed and/or no observation was present in the database. There were significant differences among subgroups of 50-60 years, 70-80 years in age; male, female in sex; Caucasian in race; small cell cancer, not otherwise specified (NOS) in histology; grade III in grade; T1a in T; NO in N; IA, IB in stage when comparing non-surgery with surgery. Significant differences were detected between 80-90 years in age and N0, N1 in N; IIA in stage when comparing sublobectomy with lobe/s (Table V). Although statistically insignificant, more patients in the following clinicopathological subgroups had survival benefits from surgery compared with non-surgery (Fig. 6): 60-70 and 80-90 years in age; Hispanic and African descent in Race; oat cell carcinoma, small cell carcinoma with intermediate cell, and 


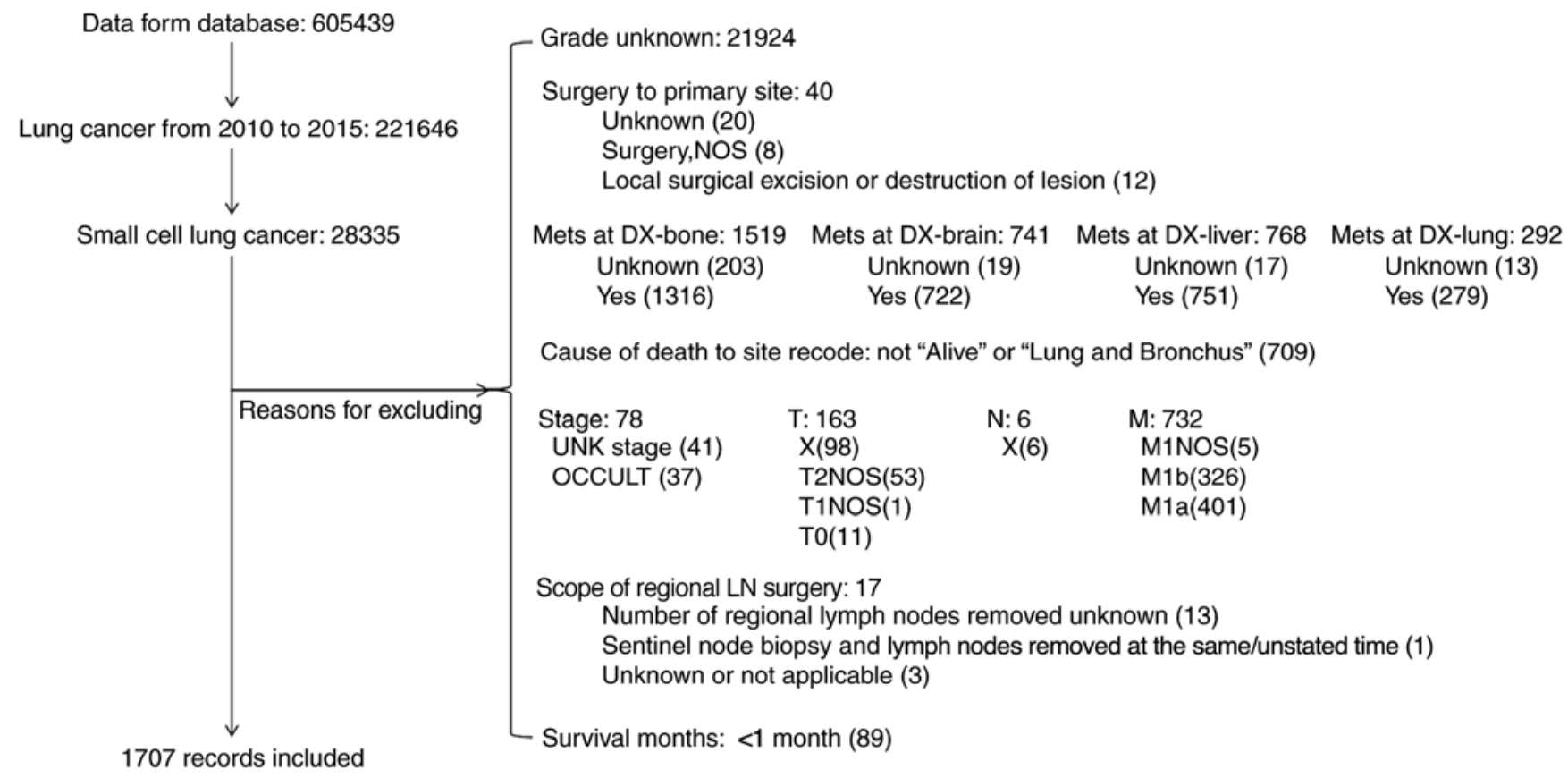

Figure 1. Diagram of data collection.
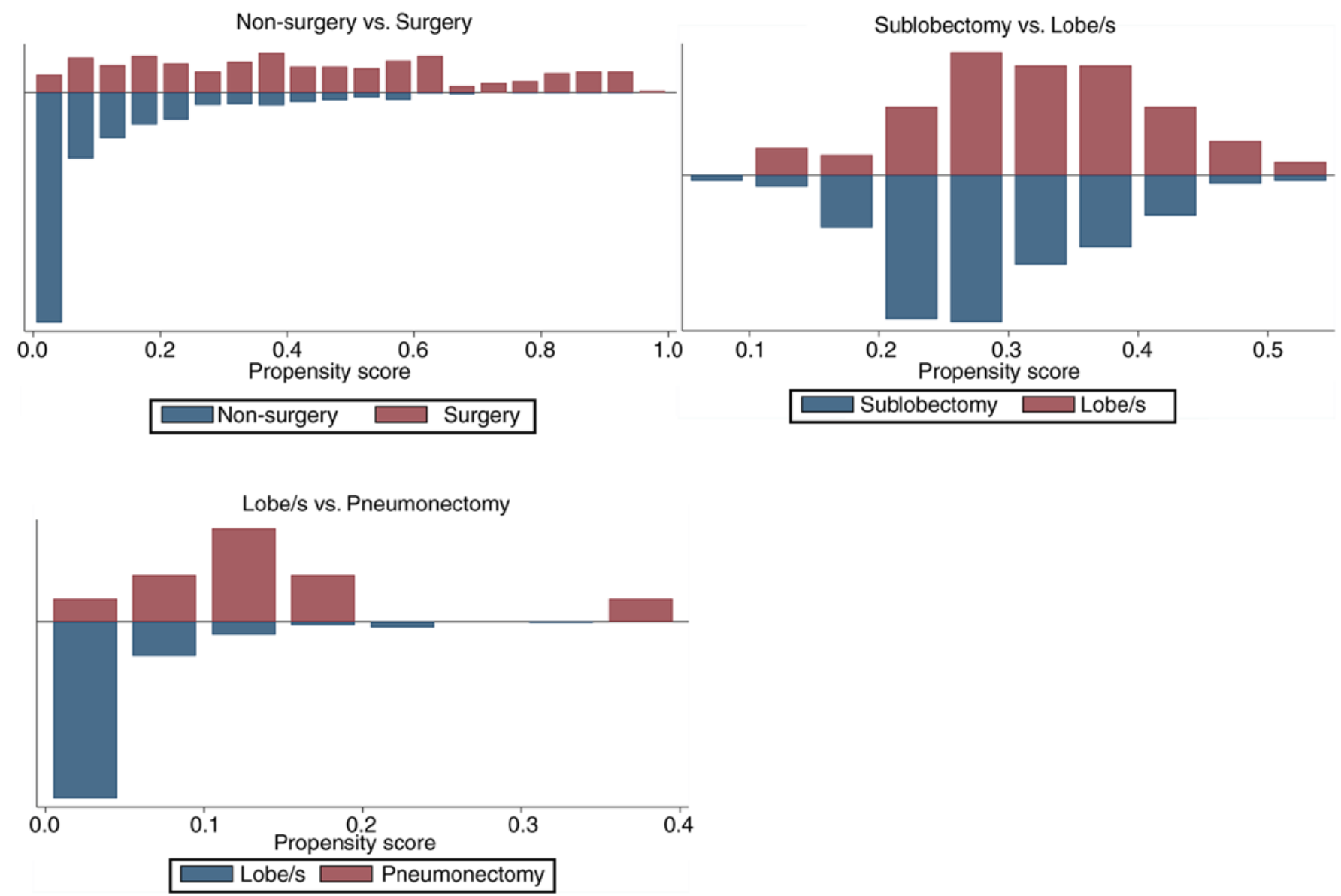

Figure 2. Histograms of propensity scores. Lobe/s, Lobectomy or bilobectomy.

combined small cell carcinoma in histology; Grade I, II, IV; $\mathrm{T} 1 \mathrm{~b}$ and T2a. Similar survival benefits in patients who received lobe/s compared with sublobectomy were observed in the following clinicopathological subgroups (Fig. 7): 60-70 years, 70-80 years in age; male and female in sex; African descent and Caucasian in race; small cell carcinoma with NOS and 
Table I. Propensity score matching test between the surgery groups.

\begin{tabular}{|c|c|c|c|c|c|c|c|c|c|}
\hline \multirow[b]{2}{*}{ Surgery } & \multirow[b]{2}{*}{ Features } & \multirow[b]{2}{*}{ Un/Matched } & \multicolumn{2}{|c|}{ Mean } & \multirow[b]{2}{*}{ \%bias } & \multirow[b]{2}{*}{ \%reduct IbiasI } & \multicolumn{2}{|c|}{ t-test } & \multirow[b]{2}{*}{$\mathrm{V}(\mathrm{T}) / \mathrm{V}(\mathrm{C})$} \\
\hline & & & Treated & Control & & & $\mathrm{t}$ & P-value & \\
\hline \multirow[t]{16}{*}{ Non-surgery vs. surgery } & \multirow[t]{2}{*}{ Sex } & $\mathrm{U}$ & 0.43197 & 0.43100 & 0.2 & & 0.03 & 0.976 & . \\
\hline & & M & 0.43197 & 0.39116 & 8.2 & $-4,086.7$ & 1.00 & 0.315 & . \\
\hline & \multirow[t]{2}{*}{ Age } & $\mathrm{U}$ & 4.19730 & 4.19670 & 0.1 & & 0.01 & 0.994 & $0.79^{\mathrm{a}}$ \\
\hline & & M & 4.19730 & 4.37070 & -17.3 & $-32,360.8$ & -2.15 & 0.032 & 0.87 \\
\hline & \multirow[t]{2}{*}{ Grade (8) } & $\mathrm{U}$ & 3.35710 & 3.56480 & -33.3 & & -5.66 & $<0.001$ & $1.63^{\mathrm{a}}$ \\
\hline & & M & 3.35710 & 3.30270 & 8.7 & 73.8 & 0.92 & 0.356 & 0.90 \\
\hline & \multirow[t]{2}{*}{ Histology } & $\mathrm{U}$ & 2.07480 & 1.16140 & 67.6 & & 14.41 & $<0.001$ & $5.82^{\mathrm{a}}$ \\
\hline & & M & 2.07480 & 1.91160 & 12.1 & 82.1 & 1.16 & 0.248 & 1.14 \\
\hline & \multirow[t]{2}{*}{ Stage (8) } & $\mathrm{U}$ & 2.75170 & 4.77570 & -131.9 & & -21.61 & $<0.001$ & $1.33^{\mathrm{a}}$ \\
\hline & & M & 2.75170 & 2.57480 & 11.5 & 91.3 & 1.29 & 0.197 & 0.96 \\
\hline & \multirow[t]{2}{*}{$\mathrm{T}(8)$} & $\mathrm{U}$ & 2.54080 & 4.29650 & -113.3 & & -17.05 & $<0.001$ & 0.80 \\
\hline & & M & 2.54080 & 2.43200 & 7.0 & 93.8 & 0.90 & 0.366 & 1.00 \\
\hline & \multirow[t]{2}{*}{$\mathrm{N}(8)$} & $\mathrm{U}$ & 0.60884 & 1.61220 & -110.9 & & -16.30 & $<0.001$ & $0.68^{\mathrm{a}}$ \\
\hline & & M & 0.60884 & 0.54082 & 7.5 & 93.2 & 1.01 & 0.313 & 0.98 \\
\hline & \multirow[t]{2}{*}{ Race } & $\mathrm{U}$ & 4.75170 & 4.68440 & 8.1 & & 1.23 & 0.217 & 0.84 \\
\hline & & $\mathrm{M}$ & 4.75170 & 4.73810 & 1.6 & 79.8 & 0.21 & 0.836 & 0.98 \\
\hline \multirow[t]{16}{*}{ Sub-lobectomy vs. lobe/s } & \multirow[t]{2}{*}{ Sex } & $\mathrm{U}$ & 0.40476 & 0.45000 & -9.1 & & -0.70 & 0.485 & . \\
\hline & & M & 0.40476 & 0.38095 & 4.8 & 47.4 & 0.31 & 0.754 & . \\
\hline & Age & $\mathrm{U}$ & 4.29760 & 4.17000 & 13.3 & & 1.06 & 0.290 & 1.36 \\
\hline & & M & 4.29760 & 4.20240 & 10.0 & 25.4 & 0.63 & 0.529 & 1.22 \\
\hline & Grade (8) & $\mathrm{U}$ & 3.40480 & 3.35000 & 7.9 & & 0.61 & 0.541 & 1.03 \\
\hline & & M & 3.40480 & 3.36900 & 5.2 & 34.8 & 0.33 & 0.742 & 0.97 \\
\hline & Histology & $\mathrm{U}$ & 1.85710 & 2.12500 & -15.6 & & -1.18 & 0.240 & 0.85 \\
\hline & & M & 1.85710 & 1.61900 & 13.8 & 11.1 & 0.99 & 0.323 & 1.29 \\
\hline & Stage (8) & $\mathrm{U}$ & 2.64290 & 2.74500 & -6.0 & & -0.48 & 0.631 & 1.37 \\
\hline & & M & 2.64290 & 2.55950 & 4.9 & 18.4 & 0.32 & 0.751 & 1.34 \\
\hline & $\mathrm{T}(8)$ & $\mathrm{U}$ & 2.22620 & 2.60500 & -25.8 & & -2.04 & 0.042 & 1.29 \\
\hline & & M & 2.22620 & 2.20240 & 1.6 & 93.7 & 0.11 & 0.916 & 1.29 \\
\hline & N (8) & $\mathrm{U}$ & 0.59524 & 0.60500 & -1.2 & & -0.09 & 0.927 & 1.43 \\
\hline & & M & 0.59524 & 0.58333 & 1.4 & -22.0 & 0.09 & 0.929 & 1.34 \\
\hline & Race & $\mathrm{U}$ & 4.83330 & 4.73500 & 13.3 & & 0.98 & 0.326 & 0.65 \\
\hline & & M & 4.83330 & 4.88100 & -6.5 & 51.6 & -0.50 & 0.616 & 1.33 \\
\hline Lobe/s vs. pneumonectomy & Sex & $\mathrm{U}$ & 0.30000 & 0.45000 & -30.6 & & -0.90 & 0.350 & . \\
\hline & & M & 0.30000 & 0.60000 & -61.1 & -100.0 & -1.34 & 0.196 & . \\
\hline & Age & $\mathrm{U}$ & 3.90000 & 4.17000 & -23.4 & & -0.90 & 0.360 & 2.42 \\
\hline & & M & 3.90000 & 4.20000 & -26.0 & -11.1 & -0.60 & 0.556 & 3.02 \\
\hline & Grade (8) & $\mathrm{U}$ & 3.10000 & 3.35000 & -31.8 & & -1.10 & 0.270 & 1.63 \\
\hline & & M & 3.10000 & 3.20000 & -12.7 & 60.0 & -0.25 & 0.806 & 0.91 \\
\hline & Histology & $\mathrm{U}$ & 2.90000 & 2.12500 & 40.6 & & 1.30 & 0.190 & 1.28 \\
\hline & & M & 2.90000 & 3.40000 & -26.2 & 35.5 & -0.55 & 0.591 & 0.96 \\
\hline & Stage (8) & $\mathrm{U}$ & 3.80000 & 2.74500 & 66.5 & & 2.10 & 0.040 & 1.09 \\
\hline & & M & 3.80000 & 3.80000 & 0.0 & 100.0 & 0.00 & 1.000 & 1.2 \\
\hline & $\mathrm{T}(8)$ & $\mathrm{U}$ & 3.90000 & 2.60500 & 87.1 & & 2.90 & $<0.001$ & 1.35 \\
\hline & & M & 3.90000 & 4.00000 & -6.7 & 92.3 & -0.14 & 0.891 & 0.95 \\
\hline & N (8) & $\mathrm{U}$ & 0.80000 & 0.60500 & 25.0 & & 0.80 & 0.440 & 1.05 \\
\hline & & M & 0.80000 & 0.80000 & 0.0 & 100.0 & 0.00 & 1.000 & 1.56 \\
\hline & Race & $\mathrm{U}$ & 4.40000 & 4.73500 & -31.5 & & -1.20 & 0.220 & 2.43 \\
\hline & & M & 4.40000 & 4.10000 & 28.2 & 10.4 & 0.53 & 0.605 & 0.97 \\
\hline
\end{tabular}

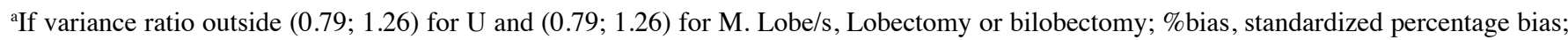
$\%$ reduct lbiasl, achieved percentage reduction in bias. 
Table II. The efficacy of the propensity score matching.

\begin{tabular}{|c|c|c|c|c|c|c|c|c|c|}
\hline Group & Sample (n) & Ps R2 & $\operatorname{LR} \chi^{2}$ & $\mathrm{P}>\chi^{2}$ & MeanBias & MedBias & $\mathrm{B}$ & $\mathrm{R}$ & $\%$ Var \\
\hline \multirow[t]{2}{*}{ Non-surgery vs. surgery } & Unmatched $(1,413)$ & 0.309 & 484.81 & $<0.01$ & 58.20 & 50.50 & $157.8^{\mathrm{a}}$ & 1.15 & 71.00 \\
\hline & Matched (294) & 0.015 & 12.59 & 0.13 & 9.30 & 8.50 & $29.4^{\mathrm{a}}$ & 1.22 & 0.00 \\
\hline \multirow[t]{2}{*}{ Sublobectomy vs. lobe/s } & Unmatched (200) & 0.030 & 10.19 & 0.25 & 11.50 & 11.20 & $41.5^{\mathrm{a}}$ & 1.09 & 0.00 \\
\hline & Matched (84) & 0.011 & 2.53 & 0.96 & 6.00 & 5.10 & 24.50 & 1.40 & 0.00 \\
\hline \multirow[t]{2}{*}{ Lobe/s vs. pneumonectomy } & Unmatched (200) & 0.161 & 12.98 & 0.11 & 42.10 & 31.70 & $111.8^{\mathrm{a}}$ & 1.78 & 0.00 \\
\hline & Matched (10) & 0.353 & 9.78 & 0.28 & 20.10 & 19.40 & $109.9^{\mathrm{a}}$ & $7.50^{*}$ & 0.00 \\
\hline
\end{tabular}

${ }^{\mathrm{a}} \mathrm{B}>25 \%$, R outside $(0.5 ; 2) ; \mathrm{B}$, absolute standardized difference of the means of the linear index of the propensity score in the unmatched and matched groups; $\mathrm{R}$, ratio of unmatched to matched variances of the propensity score index; \%Var, the percentage of continuous variables that have variance ratios that exceed the 2.5th and 97.5th percentiles of the F-distribution; Lobe/s, lobectomy or bilobectomy.
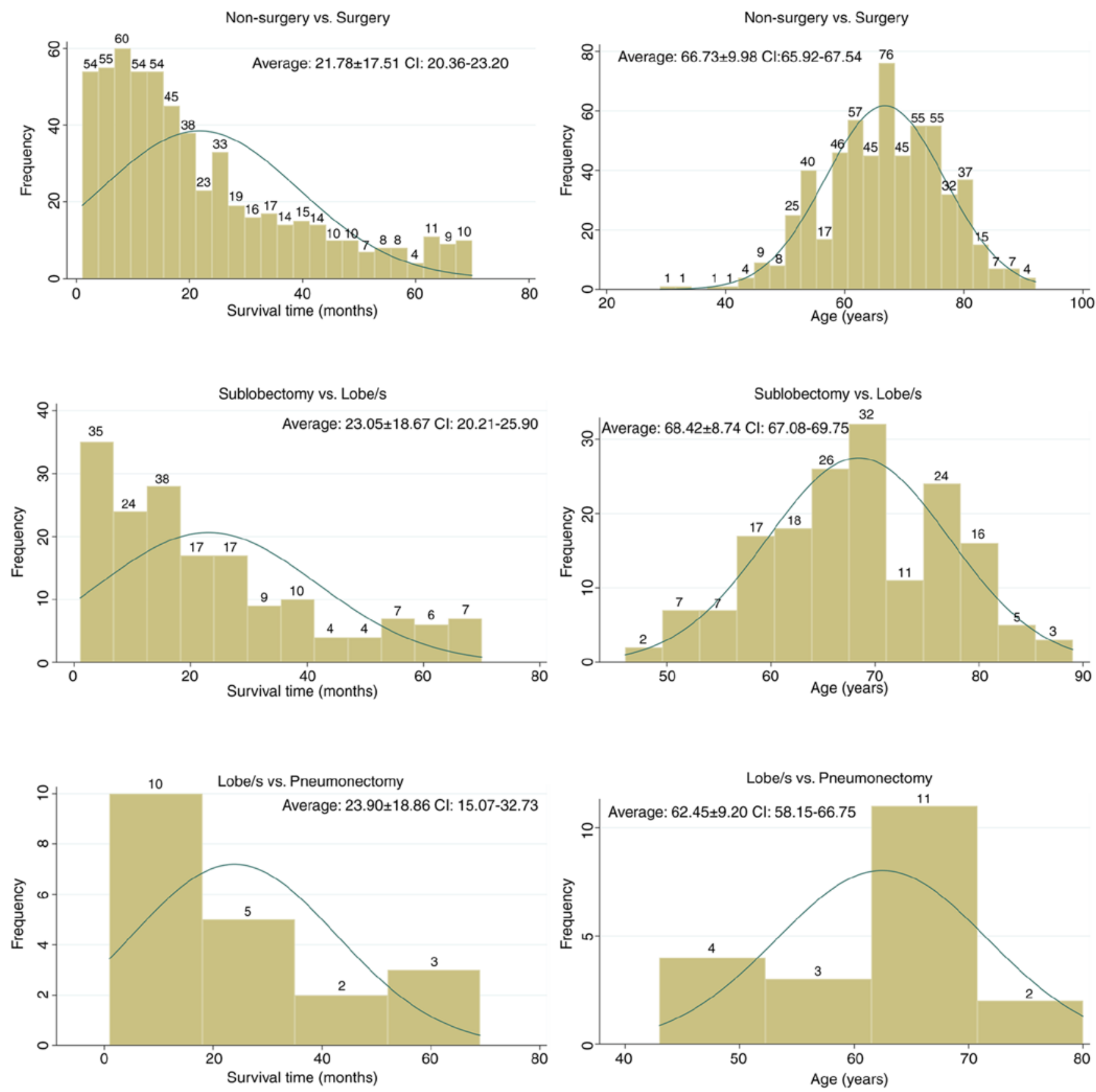

Figure 3. Distribution of survival time and age. Emerald curve, Normal-density plot. Lobe/s, Lobectomy or bilobectomy. 
Table III. Demographics of the included patients and results of univariate analysis.

A, Non-surgery vs. surgery group

\begin{tabular}{lrrrr}
\hline Features & NP & NE & NEE & P-value \\
\hline Age, years & & & & 0.341 \\
$\quad<40$ & 3 & 2 & 2.02 & \\
$\geq 40,<50$ & 22 & 10 & 12.62 & \\
$\geq 50,<60$ & 114 & 51 & 60.07 & \\
$\geq 60,<70$ & 214 & 91 & 98.62 & \\
$\geq 70,<80$ & 178 & 92 & 79.70 & \\
$\geq 80,<90$ & 53 & 28 & 21.19 & \\
$\geq 90,<100$ & 4 & 2 & 1.78 & \\
Sex & & & & 0.714 \\
Male & 252 & 118 & 120.99 & \\
Female & 336 & 158 & 155.01 & \\
Race & & & & 0.279 \\
\end{tabular}

$\begin{array}{lrrr}\text { Hispanic } & 15 & 5 & 7.69 \\ \text { AI/AN } & 2 & 0 & 0.09 \\ \text { API } & 12 & 7 & 5.15 \\ \text { African descent } & 52 & 17 & 27.18 \\ \text { Caucasian } & 506 & 247 & 235.81 \\ \text { Unknown } & 1 & 0 & 0.09 \\ \text { Histology } & & & \\ \text { SCC, NOS } & 462 & 222 & 213.83 \\ \text { OCC } & 11 & 5 & 5.69 \\ \text { SCC, IC } & 4 & 2 & 2.89 \\ \text { CSCC } & 111 & 47 & 53.59 \\ \end{array}$

Grade (8)

$\begin{array}{lrrr}\text { I } & 10 & 2 & 3.93 \\ \text { II } & 23 & 7 & 14.32 \\ \text { III } & 281 & 127 & 125.92 \\ \text { IV } & 274 & 140 & 131.83 \\ \text { T (8) } & & & \\ \text { T1a } & 189 & 86 & 92.83 \\ \text { T1b } & 128 & 60 & 63.02 \\ \text { T2a } & 173 & 77 & 79.92 \\ \text { T2b } & 29 & 19 & 10.54 \\ \text { T3 } & 53 & 25 & 21.82 \\ \text { T4 } & 16 & 9 & 7.86 \\ \text { N (8) } & & & \\ 0 & 360 & 150 & 176.48 \\ \text { 1 } & 105 & 54 & 45.92 \\ 2 & 116 & 69 & 51.28 \\ 3 & 7 & 3 & 2.33 \\ \text { Stage (8) } & & & \\ \text { IA } & 190 & 77 & 99.00 \\ \text { IB } & 112 & 44 & 53.56 \\ \text { IIA } & 108 & 56 & 45.88 \\ \text { IIB } & 31 & 13 & 13.80 \\ \text { IIIA } & 135 & 80 & 57.93 \\ \text { IIIB } & 12 & 6 & 5.84 \\ \text { Sin } & & & \end{array}$

Surgery

No

Yes
Table III. Continued.

B, Sublobectomy vs. lobe/s group

\begin{tabular}{lcccc}
\hline Features & NP & NE & NEE & P-value \\
\hline $\begin{array}{l}\text { Age, years } \\
\quad<40\end{array}$ & N/A & N/A & N/A & 0.384 \\
$\geq 40,<50$ & 2 & 2 & 0.79 & \\
$\geq 50,<60$ & 28 & 9 & 13.77 & \\
$\geq 60,<70$ & 61 & 22 & 21.40 & \\
$\geq 70,<80$ & 57 & 25 & 23.78 & \\
$\geq 80,<90$ & 20 & 8 & 6.26 & \\
$\geq 90,<100$ & N/A & N/A & N/A &
\end{tabular}

Sex

$\begin{array}{llll}\text { Male } & 56 & 25 & 22.92\end{array}$

$\begin{array}{llll}\text { Female } & 112 & 41 & 43.08\end{array}$

Race

$\begin{array}{llll}\text { Hispanic } & 2 & 1 & 0.70\end{array}$

AI/AN N/A N/A N/A

API N/A N/A N/A

$\begin{array}{llll}\text { African descent } & 8 & 4 & 4.17\end{array}$

$\begin{array}{llll}\text { Caucasian } & 157 & 61 & 61.07\end{array}$

$\begin{array}{llll}\text { Unknown } & 1 & 0 & 0.06\end{array}$

0.743

$\begin{array}{lllll}0.678 & \text { Histology } & & & \\ & \text { SCC, NOS } & 144 & 57 & 55.32\end{array}$

$\begin{array}{llll}\text { OCC } & 1 & 0 & 0.36\end{array}$

SCC, IC N/A N/A N/A

$\begin{array}{llll}\mathrm{CSCC} & 23 & 9 & 10.31\end{array}$

0.355

0.149 Grade (8)

$\begin{array}{llll}\text { I } & 4 & 0 & 1.38\end{array}$

$\begin{array}{llll}\text { II } & 5 & 1 & 2.54\end{array}$

$\begin{array}{llll}\text { III } & 72 & 27 & 29.03\end{array}$

$\begin{array}{llll}\text { IV } & 87 & 38 & 33.05\end{array}$

$0.135 \quad \mathrm{~T}(8)$

0.258

$\begin{array}{llll}\text { T1a } & 85 & 29 & 37.15\end{array}$

$\begin{array}{llll}\mathrm{T} 1 \mathrm{~b} & 27 & 11 & 11.03\end{array}$

$\begin{array}{llll}\mathrm{T} 2 \mathrm{a} & 36 & 15 & 11.00\end{array}$

$\begin{array}{llll}\mathrm{T} 2 \mathrm{~b} & 2 & 2 & 0.85\end{array}$

$\begin{array}{llll}\text { T3 } & 12 & 6 & 3.82\end{array}$

$\begin{array}{llll}\mathrm{T} 4 & 6 & 3 & 2.15\end{array}$

$0.007 \quad \mathrm{~N}(8)$

$<0.001$

$108 \quad 33 \quad 46.06$

$\begin{array}{lll}23 & 12 & 6.44\end{array}$

$35 \quad 19 \quad 13.11$

$\begin{array}{lll}2 & 2 & 0.39\end{array}$

$0.003 \quad$ Stage (8)

$\begin{array}{llll}\text { IA } & 70 & 21 & 32.86\end{array}$

$\begin{array}{llll}\text { IB } & 25 & 6 & 8.97\end{array}$

$\begin{array}{llll}\text { IIA } & 22 & 12 & 6.30\end{array}$

$\begin{array}{llll}\text { IIB } & 8 & 3 & 2.76\end{array}$

$\begin{array}{llll}\text { IIIA } & 39 & 21 & 13.51\end{array}$

$\begin{array}{llll}\text { IIIB } & 4 & 3 & 1.60\end{array}$

$<0.001 \quad$ Surgery

$\begin{array}{llll}\text { No } & 84 & 26 & 33.90\end{array}$

0.049

0.006

Yes

$84 \quad 40 \quad 32.10$ 
Table III. Continued.

C, Lobe/s vs. pneumonectomy group

\begin{tabular}{|c|c|c|c|c|c|c|c|c|c|}
\hline \multicolumn{5}{|c|}{ C, Lobe/s vs. pneumonectomy group } & Feature & NP & $\mathrm{NE}$ & NEE & P-value \\
\hline Feature & $\mathrm{NP}$ & $\mathrm{NE}$ & NEE & P-value & Surgery & & & & 0.185 \\
\hline & & & & & No & 10 & 6 & 4.02 & \\
\hline Age, years & & & & 0.210 & Yes & 10 & 4 & 5.98 & \\
\hline
\end{tabular}

$\begin{array}{lrrr}\geq 40,<50 & 2 & 1 & 1.27 \\ \geq 50,<60 & 4 & 1 & 0.85 \\ \geq 60,<70 & 10 & 8 & 4.75 \\ \geq 70,<80 & 3 & 0 & 2.44 \\ \geq 80,<90 & 1 & 0 & 0.68 \\ \geq 90,<100 & \text { N } / A & \text { N/A } & \text { N/A }\end{array}$

Sex

$\begin{array}{lrcc}\text { Male } & 6 & 3 & 3.91 \\ \text { Female } & 14 & 7 & 6.09 \\ \text { Race } & & & \\ \text { Hispanic } & 1 & 0 & 0.68 \\ \text { AI/AN } & 1 & 0 & <0.001 \\ \text { API } & 1 & 1 & 0.31 \\ \text { African descent } & 4 & 2 & 1.68 \\ \text { Caucasian } & 13 & 7 & 7.33 \\ \text { Unknown } & \text { N/A } & \text { N/A } & \text { N/A }\end{array}$

Histology

SCC, NOS

$\mathrm{OCC}$

SCC, IC

$\mathrm{CSCC}$

Grade (8)

I

II

III

IV

$\mathrm{T}(8)$

T1a

$\mathrm{T} 1 \mathrm{~b}$

$\mathrm{T} 2 \mathrm{a}$

$\mathrm{T} 2 \mathrm{~b}$

T3

$\mathrm{T} 4$

$\mathrm{N}(8)$

0

1

2

3

Stage (8)

IA

IB

IIA

IIB

IIIA

IIIB
Table III. Continued.

Lobe/s, lobectomy or bilobectomy; NP, Number of Patients; NE, Number of Events; NEE, Number of Expected Events; AI/AN, American Indian/Alaska Native; API, Asian or Pacific Islander; SCC, Small cell carcinoma; OCC, Oat cell carcinoma; IC, Intermediate cell; CSCC, Combined small cell carcinoma; N/A, No observation in the database.

0.544

combined small cell carcinoma in histology; Grade III and IV; T1a and T1b. Generally, patients who did not receive surgery $(\mathrm{P}<0.001)$ or received sublobectomy $(\mathrm{P}=0.03)$ were at an increased risk of mortality when compared with patients who received surgery or lobe/s respectively (Table VI). Fig. 8 shows the cumulative survival curves of each group.

\section{Discussion}

As SCLC responds to chemotherapy and radiotherapy, surgical treatment is considered to be an option for stage I-IIA (T1-2, N0, M0) SCLC $(9,10)$. The most recent National Comprehensive Cancer Network (NCCN) guidelines recommend that patients with SCLC at clinical stage I-IIA (T1-2, N0, M0) after a standard staging evaluation may be eligible for surgical resection (9). After analyzing the SEER database, Schreiber et al (11) concluded that the use of surgery, and particularly lobectomy, in selected patients with limited-stage SCLC was associated with improved survival outcomes. However, there was inherent selection bias in their study (11). Therefore, the present study performed the period propensity score matching analysis using the SEER database to further examine the role of surgery on survival in patients with SCLC.

Following propensity score matching analysis, the present study identified that the clinicopathological features of $\mathrm{N}$ stage and surgery were important factors for postoperative CSS in patients with SCLC who received surgery, including sublobectomy, lobe/s and pneumonectomy. This finding was corroborated by the results of previous studies $(5,11,12)$. The IASLC proposals $(12)$ demonstrated that there was a significant difference in the survival of patients who underwent surgery between N0 patients and those with node-positive disease for both clinical and pathological staging, independent of $\mathrm{T}$ category. Takenaka et al (5) reported that the 5-year survival rates of the patients with SCLC with or without surgical resection, according to the clinical stage were as follows: 62 and $25 \%$ in stage I $(\mathrm{P}<0.01), 33$ and $24 \%$ in stage II $(\mathrm{P}=0.95)$ and 18 and $18 \%$ in stage III $(\mathrm{P}=0.35)$. The study of Schreiber et al (11) also demonstrated that the overall survival for patients with SCLC with N0, N1 and N2 who received surgery were significantly improved, when compared with those who did not receive surgery $(\mathrm{P}<0.01)$. In addition, the significance of surgery was corroborated following Cox regression analysis $(\mathrm{P}<0.001)$ (Table IV). These results suggested that the role of surgery for patients with SCLC was significant. 
A Group A: Non-surgery vs. surgery

N

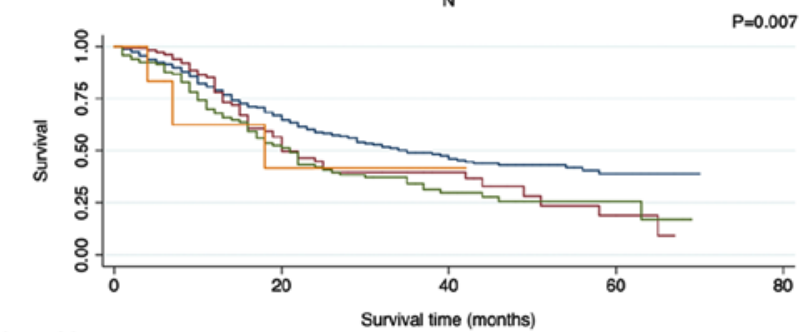

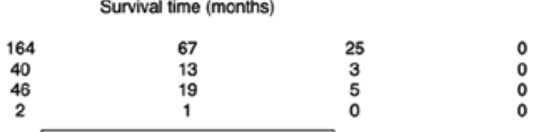

B
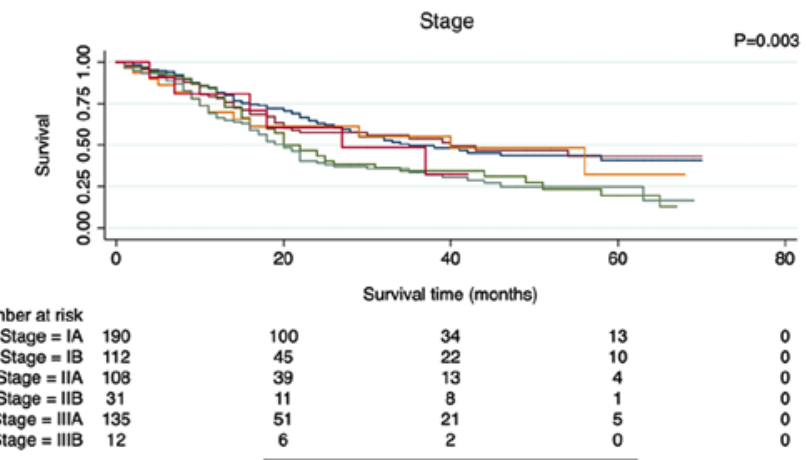

C

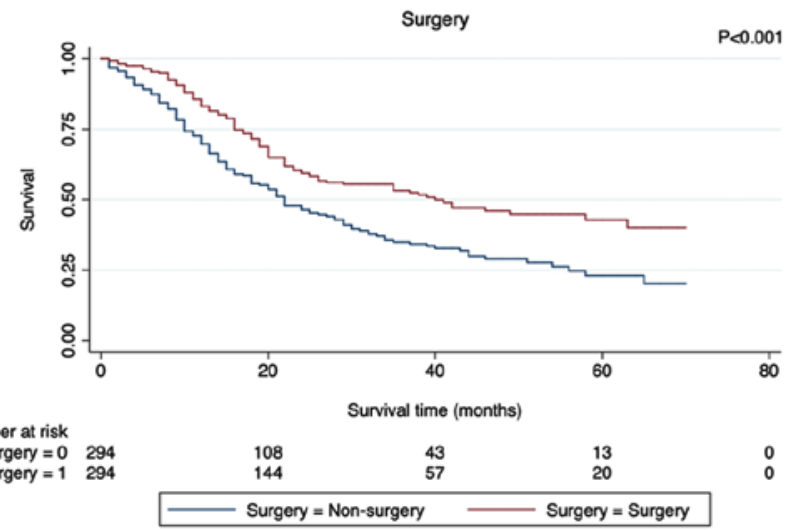

D Group B: Sublobectomy vs. Lobe/s

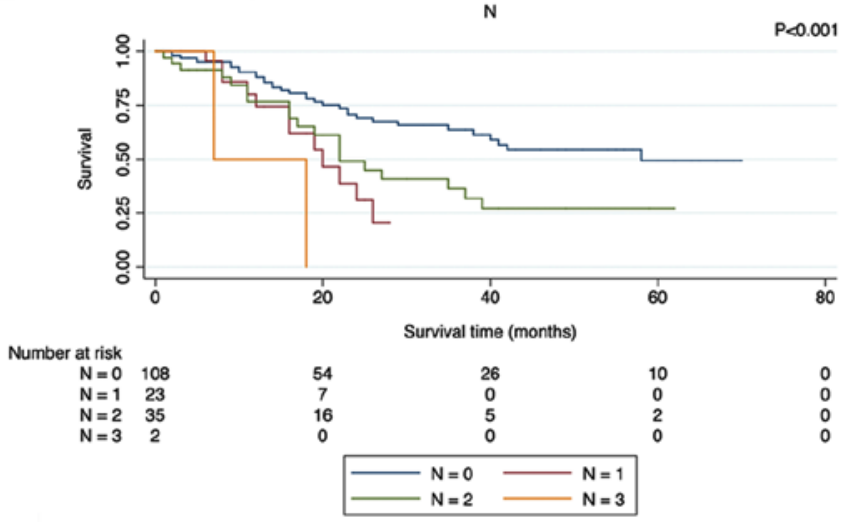

E

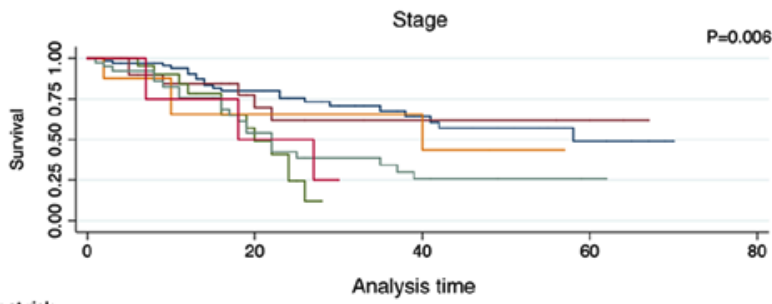

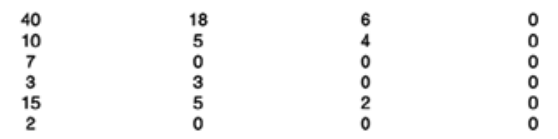

$\mathbf{F}$

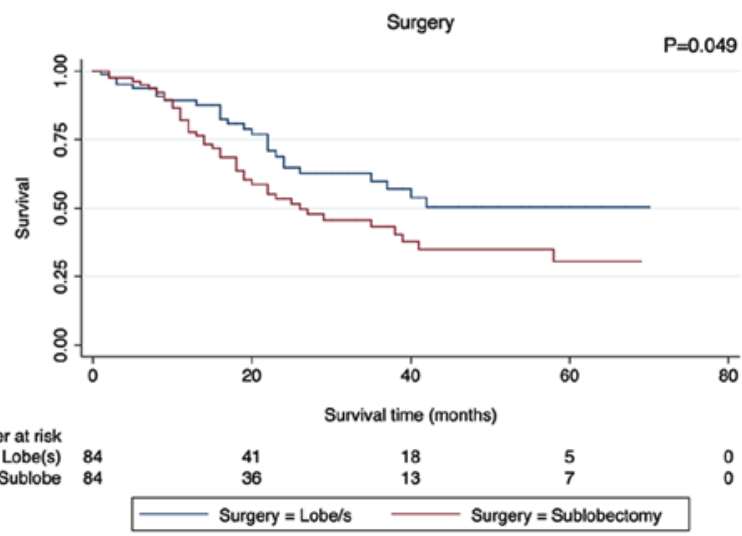

Figure 4. Potential prognostic factors analyzed by Kaplan-Meier. (A) N stage, (B) stage and (C) surgery comparing non-surgery with surgery and (D) $\mathrm{N}$ stage, (E) stage and (F) surgery comparing sublobectomy with lobe/s. Lobe/s, lobectomy or bilobectomy.

To further identify who would benefit from surgery, the survival functions of surgery stratified by the clinicopathological features were analyzed using log-rank tests (Table V). The results revealed that patients who did not receive surgery in any of the subgroups, including sex, histology and grade had an increased risk of COD compared with patients who received surgery. These results suggest that surgery should be performed irrespective of sex, histology, grade and clinicopathological features. Previous studies have reported that increasing age was an independent adverse prognostic factor in SCLC (13-15). However, the results of the present study demonstrated that patients between 50 and 90 years of age benefited from surgery, although analysis was not tested in the subgroups of age $<40$ years or between 90 and 100 years. Furthermore, age was an independent prognostic factor $(\mathrm{P}<0.001$; Table IV), which suggested that surgery should also be performed even in elderly patients with SCLC. This result was similar with the treatment of thoracic irradiation for limited-stage SCLC, in which it was reported that in the dose range examined, age did not appear to have an effect on the delivery, tolerance or efficacy of TI in the combined modality management of SCLC (16). Concerning T, there was significant difference in the T1a subgroup $(\mathrm{P}<0.001)$ and better survival trends in the T1b and T2a subgroups. Furthermore, patients 
Table IV. Multivariate analysis of different surgery types.

A, Non-surgery vs. surgery group

\begin{tabular}{lccr}
\hline & \multicolumn{3}{c}{ Multivariate analysis } \\
\cline { 2 - 4 } Characteristic & Hazard ratio & 95\% Confidence \\
interval & P-value \\
\hline Age & 1.21 & $1.07-1.36$ & $<0.01$ \\
Sex & 0.97 & $0.76-1.23$ & 0.78 \\
Race & 1.11 & $0.92-1.35$ & 0.27 \\
Histology & 0.98 & $0.90-1.06$ & 0.60 \\
Grade & 1.11 & $0.91-1.36$ & 0.29 \\
T & 1.13 & $0.98-1.30$ & 0.09 \\
N & 1.27 & $0.85-1.88$ & 0.24 \\
Stage & 1.01 & $0.81-1.27$ & 0.92 \\
Surgery & 0.59 & $0.47-0.76$ & $<0.01$ \\
\hline
\end{tabular}

B, Sublobectomy vs. lobe/s group

\begin{tabular}{lccc}
\hline & \multicolumn{3}{c}{ Multivariate analysis } \\
\cline { 2 - 4 } Characteristic & Hazard ratio & 95\% Confidence \\
interval & P-value \\
\hline Age & 1.23 & $0.94-1.61$ & 0.92 \\
Sex & 1.03 & $0.61-1.71$ & 0.92 \\
Race & 0.84 & $0.51-1.40$ & 0.51 \\
Histology & 0.99 & $0.81-1.20$ & 0.91 \\
Grade & 1.64 & $1.03-2.60$ & 0.29 \\
T & 1.27 & $1.00-1.63$ & 0.05 \\
N & 2.04 & $0.90-4.64$ & 0.09 \\
Stage & 0.83 & $0.52-1.32$ & 0.92 \\
Surgery & 1.67 & $0.96-2.90$ & 0.07 \\
\hline
\end{tabular}

C, Lobe/s vs. Pneumonectomy group

\begin{tabular}{lccc}
\hline & \multicolumn{3}{c}{ Multivariate analysis } \\
\cline { 2 - 4 } Characteristic & Hazard ratio & 95\% Confidence \\
interval & P-value \\
\hline Age & 0.28 & $0.05-1.47$ & 0.13 \\
Sex & 0.22 & $0.03-1.58$ & 0.13 \\
Race & 0.20 & $0.03-1.59$ & 0.51 \\
Histology & 2.47 & $0.93-6.52$ & 0.07 \\
Grade & 0.77 & $0.18-3.37$ & 0.73 \\
T & 2.80 & $0.20-39.85$ & 0.45 \\
N & 0.86 & $0.13-5.87$ & 0.88 \\
Stage & 2.06 & $0.12-34.16$ & 0.61 \\
Surgery & 2.39 & $0.18-32.14$ & 0.51 \\
\hline
\end{tabular}

Lobe/s, lobectomy or bilobectomy.

with SCLC with N0 $(\mathrm{P}<0.001)$ and stage Ia $(\mathrm{P}<0.001)$ and Ib $(\mathrm{P}<0.001)$ would have an increased benefit from surgery. These results, which were in accordance with those reported by the IASLC (12), clarified why the most recent NCCN guidelines
Table V. Survival functions of clinicopathological features stratified by surgery.

A. Non-surgery vs. surgery group

\begin{tabular}{|c|c|c|c|c|c|}
\hline \multirow[b]{2}{*}{ Feature } & \multicolumn{2}{|c|}{ Non-surgery } & \multicolumn{2}{|c|}{ Surgery } & \multirow[b]{2}{*}{ P-value } \\
\hline & $\mathrm{NE}$ & NEE & $\mathrm{NE}$ & NEE & \\
\hline Age, years & & & & & $<0.01$ \\
\hline$<40$ & 2 & 2.0 & $\mathrm{NT}$ & NT & NS \\
\hline$\geq 40,<50$ & 6 & 6.2 & 4 & 3.8 & 0.91 \\
\hline$\geq 50,<60$ & 33 & 20.8 & 18 & 30.2 & $<0.01$ \\
\hline$\geq 60,<70$ & 46 & 39.6 & 45 & 51.4 & 0.17 \\
\hline$\geq 70,<80$ & 53 & 39.8 & 39 & 52.2 & $<0.01$ \\
\hline$\geq 80,<90$ & 19 & 15.5 & 9 & 12.5 & 0.17 \\
\hline$\geq 90,<100$ & 2 & 2.0 & NT & NT & NS \\
\hline Sex & & & & & $<0.01$ \\
\hline Male & 65 & 54.0 & 53 & 64.0 & 0.04 \\
\hline Female & 96 & 71.3 & 62 & 86.7 & $<0.01$ \\
\hline Race & & & & & $<0.01$ \\
\hline Hispanic & 2 & 1.9 & 3 & 3.1 & 0.89 \\
\hline $\mathrm{AI} / \mathrm{AN}$ & NT & NT & $\mathrm{NT}$ & NT & NS \\
\hline API & 3 & 3.1 & 4 & 3.9 & 0.91 \\
\hline African descent & 9 & 8.1 & 8 & 8.9 & 0.66 \\
\hline Caucasian & 147 & 111.3 & 100 & 135.7 & $<0.01$ \\
\hline Histology & & & & & $<0.01$ \\
\hline SCC, NOS & 139 & 113.5 & 83 & 108.5 & $<0.01$ \\
\hline OCC & 5 & 4.2 & 0 & 0.8 & 0.29 \\
\hline $\mathrm{SCC}, \mathrm{IC}$ & 2 & 0.8 & 0 & 1.2 & 0.09 \\
\hline CSCC & 15 & 9.7 & 32 & 37.3 & 0.05 \\
\hline Grade (8) & & & & & $<0.01$ \\
\hline I & 1 & 0.5 & 1 & 1.5 & 0.37 \\
\hline II & 3 & 2.0 & 4 & 5.0 & 0.42 \\
\hline III & 77 & 55.6 & 50 & 71.4 & $<0.01$ \\
\hline IV & 80 & 69.5 & 60 & 70.5 & 0.07 \\
\hline $\mathrm{T}(8)$ & & & & & $<0.01$ \\
\hline T1a & 56 & 34.5 & 30 & 51.5 & $<0.01$ \\
\hline $\mathrm{T} 1 \mathrm{~b}$ & 42 & 36.8 & 18 & 23.2 & 0.16 \\
\hline $\mathrm{T} 2 \mathrm{a}$ & 38 & 29.7 & 39 & 47.4 & 0.05 \\
\hline $\mathrm{T} 2 \mathrm{~b}$ & 15 & 15.1 & 4 & 3.9 & 0.96 \\
\hline $\mathrm{T} 3$ & 7 & 9.1 & 18 & 15.9 & 0.36 \\
\hline $\mathrm{T} 4$ & 3 & 2.5 & 6 & 6.5 & 0.68 \\
\hline $\mathrm{N}(8)$ & & & & & $<0.01$ \\
\hline 0 & 100 & 68.6 & 50 & 81.4 & $<0.01$ \\
\hline 1 & 22 & 24.3 & 32 & 29.7 & 0.49 \\
\hline 2 & 38 & 32.2 & 31 & 36.8 & 0.15 \\
\hline 3 & 1 & 1.8 & 2 & 1.2 & 0.31 \\
\hline Stage (8) & & & & & $<0.01$ \\
\hline IA & 50 & 31.1 & 27 & 45.9 & $<0.01$ \\
\hline IB & 31 & 20.8 & 13 & 23.2 & $<0.01$ \\
\hline IIA & 32 & 32.0 & 24 & 24.0 & 1.00 \\
\hline IIB & 7 & 7.2 & 6 & 5.9 & 0.93 \\
\hline IIIA & 39 & 33.5 & 41 & 46.5 & 0.20 \\
\hline IIIB & 2 & 2.7 & 4 & 3.3 & 0.58 \\
\hline
\end{tabular}


Table V. Continued.

B, Sublobectomy vs. lobe/s group

\begin{tabular}{|c|c|c|c|c|c|}
\hline \multirow[b]{2}{*}{ Feature } & \multicolumn{2}{|c|}{$\begin{array}{c}\text { Sub- } \\
\text { lobectomy }\end{array}$} & \multicolumn{2}{|c|}{ lobe/s } & \multirow[b]{2}{*}{ P-value } \\
\hline & $\mathrm{NE}$ & NEE & $\mathrm{NE}$ & NEE & \\
\hline Age, years & & & & & 0.05 \\
\hline$<40$ & $\mathrm{~N} / \mathrm{A}$ & $\mathrm{N} / \mathrm{A}$ & N/A & $\mathrm{N} / \mathrm{A}$ & $\mathrm{NS}$ \\
\hline$\geq 40,<50$ & 2 & 2.0 & NT & NT & NS \\
\hline$\geq 50,<60$ & 5 & 5.5 & 4 & 3.5 & 0.75 \\
\hline$\geq 60,<70$ & 12 & 9.6 & 10 & 12.4 & 0.30 \\
\hline$\geq 70,<80$ & 14 & 11.3 & 11 & 13.7 & 0.28 \\
\hline$\geq 80,<90$ & 7 & 4.0 & 1 & 4.1 & 0.03 \\
\hline$\geq 90,<100$ & N/A & N/A & N/A & N/A & NS \\
\hline Sex & & & & & 0.06 \\
\hline Male & 17 & 14.9 & 8 & 10.1 & 0.38 \\
\hline Female & 23 & 17.7 & 18 & 23.3 & 0.09 \\
\hline Race & & & & & 0.05 \\
\hline Hispanic & 1 & 1.0 & NT & NT & NS \\
\hline $\mathrm{AI} / \mathrm{AN}$ & $\mathrm{N} / \mathrm{A}$ & N/A & N/A & N/A & N/A \\
\hline API & $\mathrm{N} / \mathrm{A}$ & $\mathrm{N} / \mathrm{A}$ & N/A & $\mathrm{N} / \mathrm{A}$ & NS \\
\hline African descent & 3 & 2.8 & 1 & 1.2 & 0.81 \\
\hline Caucasian & 36 & 28.4 & 25 & 32.6 & 0.05 \\
\hline Histology & & & & & 0.04 \\
\hline SCC, NOS & 32 & 25.1 & 25 & 31.9 & 0.06 \\
\hline $\mathrm{OCC}$ & NT & NT & NT & NT & NS \\
\hline $\mathrm{SCC}, \mathrm{IC}$ & $\mathrm{N} / \mathrm{A}$ & N/A & N/A & $\mathrm{N} / \mathrm{A}$ & NS \\
\hline CSCC & 8 & 6.8 & 1 & 2.2 & 0.36 \\
\hline Grade (8) & & & & & 0.06 \\
\hline I & NT & NT & NT & NT & NS \\
\hline II & 1 & 1.0 & 0 & 0.0 & 1.00 \\
\hline III & 18 & 14.5 & 9 & 12.5 & 0.17 \\
\hline IV & 21 & 17.0 & 17 & 21.0 & 0.19 \\
\hline $\mathrm{T}(8)$ & & & & & 0.07 \\
\hline T1a & 14 & 10.7 & 7 & 10.4 & 0.52 \\
\hline $\mathrm{T} 1 \mathrm{~b}$ & 5 & 3.5 & 1 & 2.5 & 0.48 \\
\hline $\mathrm{T} 2 \mathrm{a}$ & 5 & 1.0 & 7 & 11.0 & 0.22 \\
\hline $\mathrm{T} 2 \mathrm{~b}$ & 1 & 0.6 & 2 & 2.4 & 0.32 \\
\hline $\mathrm{T} 3$ & 12 & 12.6 & 9 & 8.4 & 0.24 \\
\hline $\mathrm{T} 4$ & 3 & 3.0 & NT & NT & NS \\
\hline $\mathrm{N}(8)$ & & & & & 0.02 \\
\hline 0 & 22 & 16.2 & 11 & 16.8 & 0.04 \\
\hline 1 & 5 & 1.2 & 7 & 10.8 & $<0.001$ \\
\hline 2 & 11 & 12.2 & 8 & 6.8 & 0.56 \\
\hline 3 & 2 & 2.0 & NT & NT & NS \\
\hline Stage (8) & & & & & 0.02 \\
\hline IA & 14 & 10.7 & 7 & 10.4 & 0.14 \\
\hline IB & 5 & 3.5 & 1 & 2.5 & 0.20 \\
\hline IIA & 5 & 1.0 & 7 & 11.0 & $<0.01$ \\
\hline IIB & 1 & 0.6 & 2 & 2.4 & 0.56 \\
\hline IIIA & 12 & 12.6 & 9 & 8.4 & 0.77 \\
\hline IIIB & 3 & 3.0 & NT & NT & NS \\
\hline
\end{tabular}

Table V. Continued.

C, Lobe/s vs. Pneumonectomy group

\begin{tabular}{|c|c|c|c|c|c|}
\hline \multirow[b]{2}{*}{ Feature } & \multicolumn{2}{|c|}{ lobe/s } & \multicolumn{2}{|c|}{$\begin{array}{l}\text { Pneumonec- } \\
\text { tomy }\end{array}$} & \multirow[b]{2}{*}{ P-value } \\
\hline & $\mathrm{NE}$ & NEE & $\mathrm{NE}$ & NEE & \\
\hline Age, years & & & & & 0.42 \\
\hline$<40$ & N/A & N/A & N/A & $\mathrm{N} / \mathrm{A}$ & NS \\
\hline$\geq 40,<50$ & NT & NT & 1 & 1.0 & NS \\
\hline$\geq 50,<60$ & 0 & 0.0 & 1 & 1.0 & 1.00 \\
\hline$\geq 60,<70$ & 6 & 6.7 & 2 & 1.3 & 0.42 \\
\hline$\geq 70,<80$ & NT & NT & NT & NT & NS \\
\hline$\geq 80,<90$ & NT & NT & NT & NT & NS \\
\hline$\geq 90,<100$ & N/A & N/A & N/A & N/A & $\mathrm{NS}$ \\
\hline Sex & & & & & 0.18 \\
\hline Male & 2 & 1.2 & 1 & 1.9 & 0.3 \\
\hline Female & 4 & 2.8 & 3 & 4.2 & 0.35 \\
\hline Race & & & & & 0.24 \\
\hline Hispanic & NT & NT & NT & NT & NS \\
\hline $\mathrm{AI} / \mathrm{AN}$ & NT & NT & NT & NT & NS \\
\hline API & 1 & 1.0 & N/A & $\mathrm{N} / \mathrm{A}$ & NS \\
\hline African descent & 1 & 0.5 & 1 & 1.5 & 0.32 \\
\hline Caucasian & 4 & 2.9 & 3 & 4.1 & 0.39 \\
\hline Histology & & & & & 0.70 \\
\hline $\mathrm{SCC}, \mathrm{NOS}$ & 1 & 0.2 & 1 & 1.8 & 0.03 \\
\hline OCC & N/A & N/A & N/A & N/A & NS \\
\hline $\mathrm{SCC}, \mathrm{IC}$ & NT & NT & NT & NT & NS \\
\hline CSCC & 5 & 5.3 & 3 & 2.7 & 0.80 \\
\hline Grade (8) & & & & & 0.20 \\
\hline I & 1 & 1.0 & NT & NT & NS \\
\hline II & 1 & 0.8 & 1 & 1.3 & 0.71 \\
\hline III & 2 & 1.7 & 1 & 1.3 & 0.70 \\
\hline IV & 2 & 0.9 & 2 & 3.1 & 0.14 \\
\hline $\mathrm{T}(8)$ & & & & & 0.31 \\
\hline T1a & NT & NT & NT & NT & NS \\
\hline $\mathrm{T} 1 \mathrm{~b}$ & N/A & N/A & N/A & N/A & NS \\
\hline $\mathrm{T} 2 \mathrm{a}$ & 1 & 0.2 & 1 & 1.8 & 0.05 \\
\hline $\mathrm{T} 2 \mathrm{~b}$ & 0 & 0.0 & 1 & 1.0 & 1.00 \\
\hline T3 & 3 & 2.9 & 1 & 1.1 & 0.91 \\
\hline $\mathrm{T} 4$ & 2 & 1.7 & 1 & 1.3 & 0.69 \\
\hline N (8) & & & & & 0.24 \\
\hline 0 & 0 & 0.5 & 2 & 1.6 & 0.45 \\
\hline 1 & 4 & 2.1 & 1 & 2.9 & 0.06 \\
\hline 2 & 2 & 1.8 & 1 & 1.2 & 0.78 \\
\hline 3 & N/A & N/A & N/A & $\mathrm{N} / \mathrm{A}$ & NS \\
\hline Stage (8) & & & & & 0.48 \\
\hline IA & NT & NT & NT & NT & NS \\
\hline IB & 1 & 1.0 & N/A & N/A & NS \\
\hline IIA & 1 & 0.5 & 0 & 0.5 & 0.32 \\
\hline IIB & NT & NT & NT & NT & NS \\
\hline IIIA & 4 & 3.6 & 3 & 3.4 & 0.74 \\
\hline IIIB & 1 & 1.0 & NT & NT & NS \\
\hline
\end{tabular}

Lobe/s, lobectomy or bilobectomy; NP, Number of Patients; NE, Number of Events; NEE, Number of Expected Events; AI/AN, American Indian/Alaska Native; API, Asian or Pacific Islander; SCC, Small cell carcinoma; OCC, Oat cell carcinoma; IC, Intermediate cell; CSCC, Combined small cell carcinoma; NT, No test possible because of no failures observed; N/A, No observation in the database. 

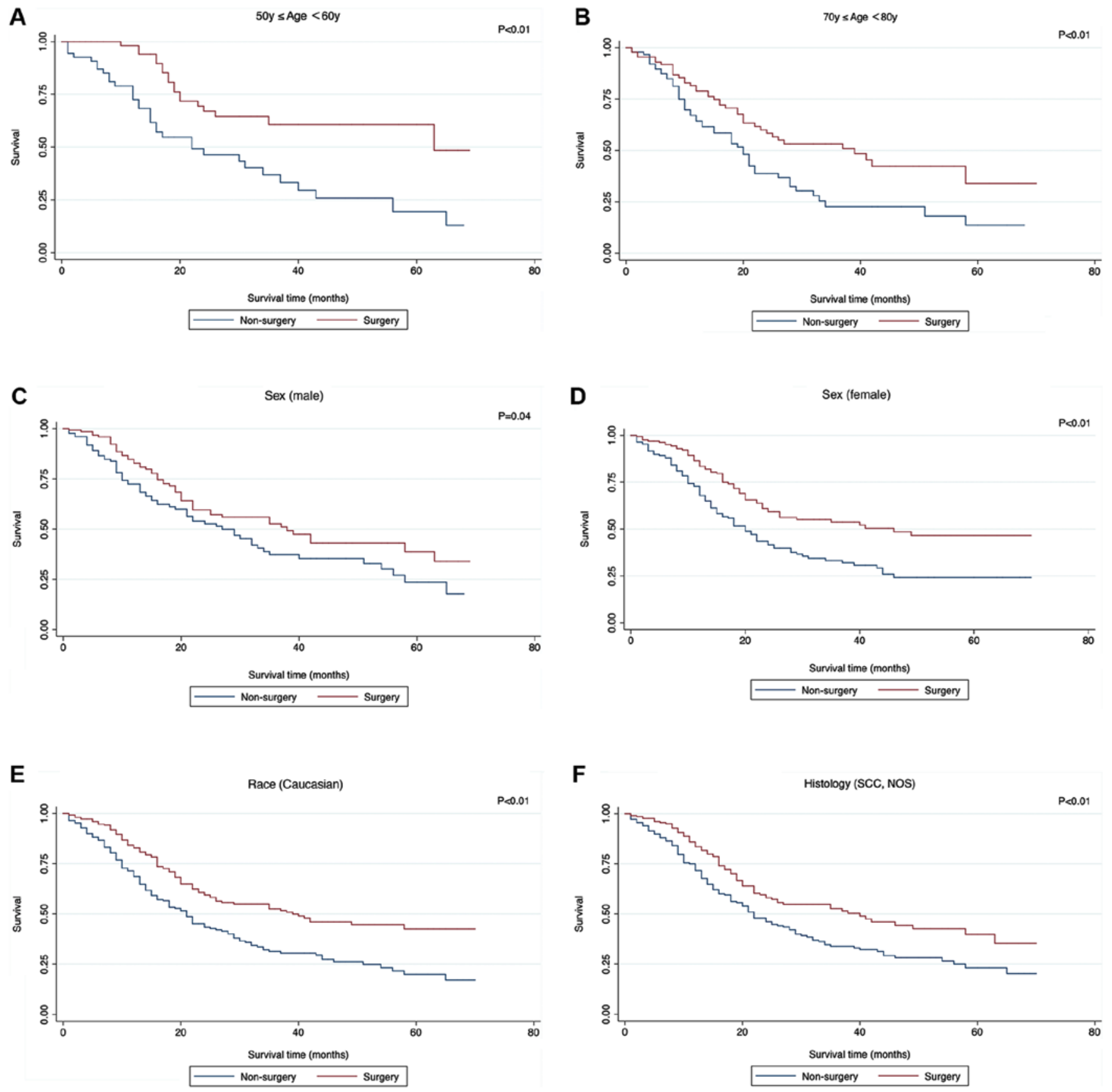

Figure 5. Significant survival functions of surgery stratified by clinicopathological features when comparing Non-surgery with surgery. (A) Age, $\geq 60$ and $<70$ years; (B) age, $\geq 70$ and <80 years; (C) sex, male; (D), sex, female; (E) race, Caucasian; (F) histology, SCC, NOS; SCC, small cell cancer; NOS, not otherwise specified.

recommend that patients with SCLC with clinical stage I-IIA (T1-2, N0, M0) after a standard staging evaluation may be considered for surgical resection (9).

As with the comparison of non-surgery with surgery, following propensity score matching analysis, the present study identified that the clinicopathological features of $\mathrm{N}$, stage and surgery were important factors in postoperative CSS in patients with SCLC who received sublobectomy compared with those who received lobe/s. However, no independent prognostic factor was identified in the Cox regression model.

When the survival functions of surgery stratified by clinicopathological features were analyzed using log-rank tests (Table V), more patients who received sublobectomy in all subgroups of sex, histology were at risk of COD compared with those who received lobe/s. On the other hand, the results also demonstrated that there were more patients between 60 and 90 years of age, who benefited from lobe/s, although this analysis was not performed in the subgroups ages $<50$ years, as no failure events were observed, and in the subgroups ages $<40$ years and between 90 and 100 years, due to the absence of observations. Another study comparing treatment strategies for stage I SCLC using the National Cancer Database (17) demonstrated that lobectomy was associated with an improved survival compared with limited resection (HR 0.64; 95\% CI, 0.53-0.78; P<0.001). Schreiber et al (11) revealed that the median survival time for lobectomy and sublobectomy was 40 and 23 months, respectively 
Table VI. The incidence of cause of death to site.

\begin{tabular}{lrrrr}
\hline Surgery & Alive & Death & Total & P \\
\hline Non-surgery & 133 & 161 & 294 & $<0.01^{\text {a }}$ \\
Surgery & 179 & 115 & 294 & $0.03^{\text {a }}$ \\
Sublobectomy & 44 & 40 & 84 & 0.37 \\
Lobectomy or bilobectomy & 58 & 26 & 84 & 10 \\
Lobectomy or bilobectomy & 4 & 6 & 10 & \\
Pneumonectomy & 6 & 4 & & \\
\hline
\end{tabular}

${ }^{\mathrm{a}} \mathrm{P}<0.05$.
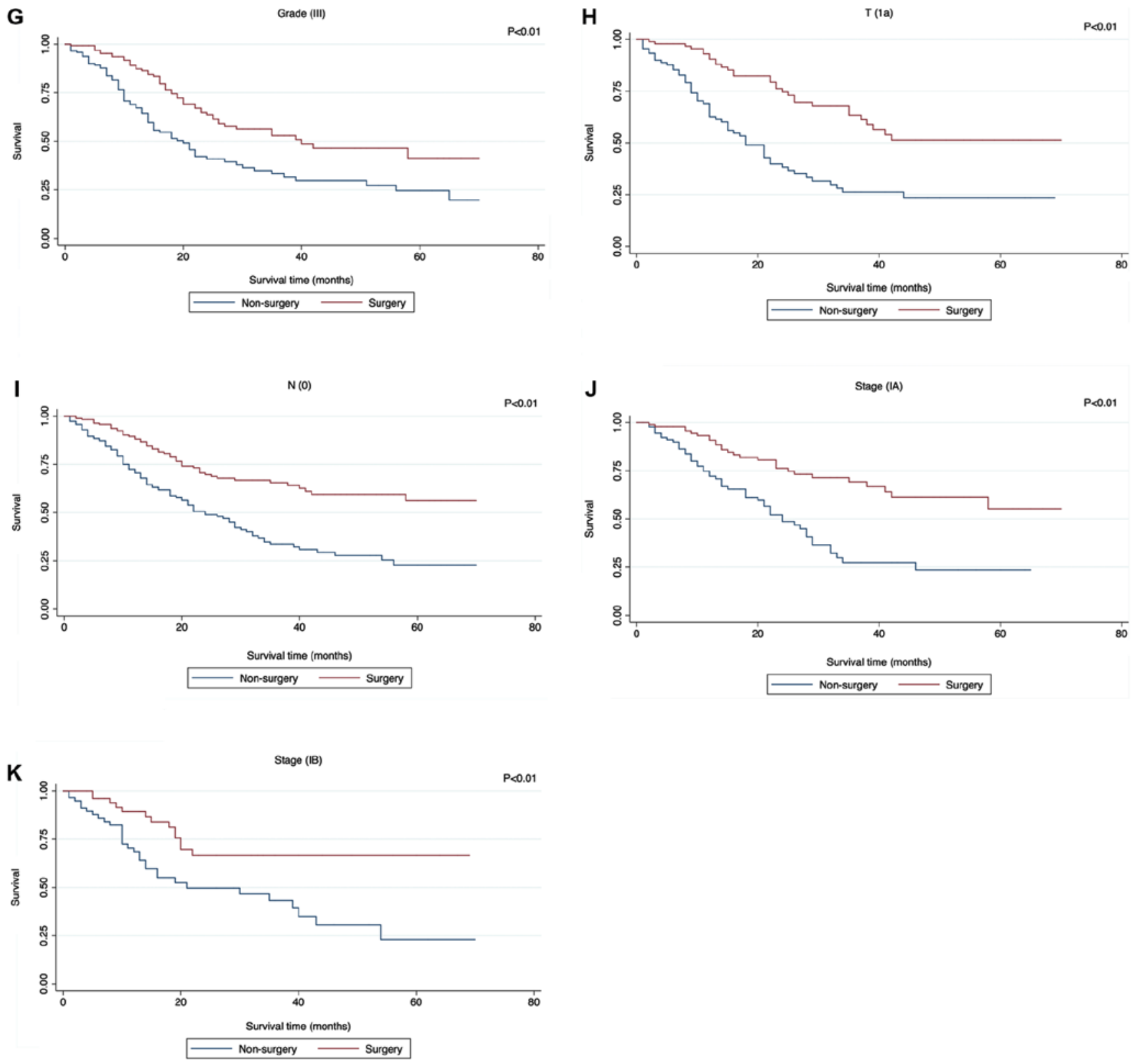

Figure 5. Continued. (G) grade III; (H) T1a; (I) N0; (J) Stage IA; (K) Stage IB. SCC, small cell cancer; NOS, not otherwise specified.

$(\mathrm{P}<001)$. These results confirmed that, similar with the NCCN recommendation (9), bi-/lobecotomy was the preferred operation for SCLC compared with sublobectomy, even in elder patients irrespective of sex and histology. When analyzing $\mathrm{T}$, although 
A
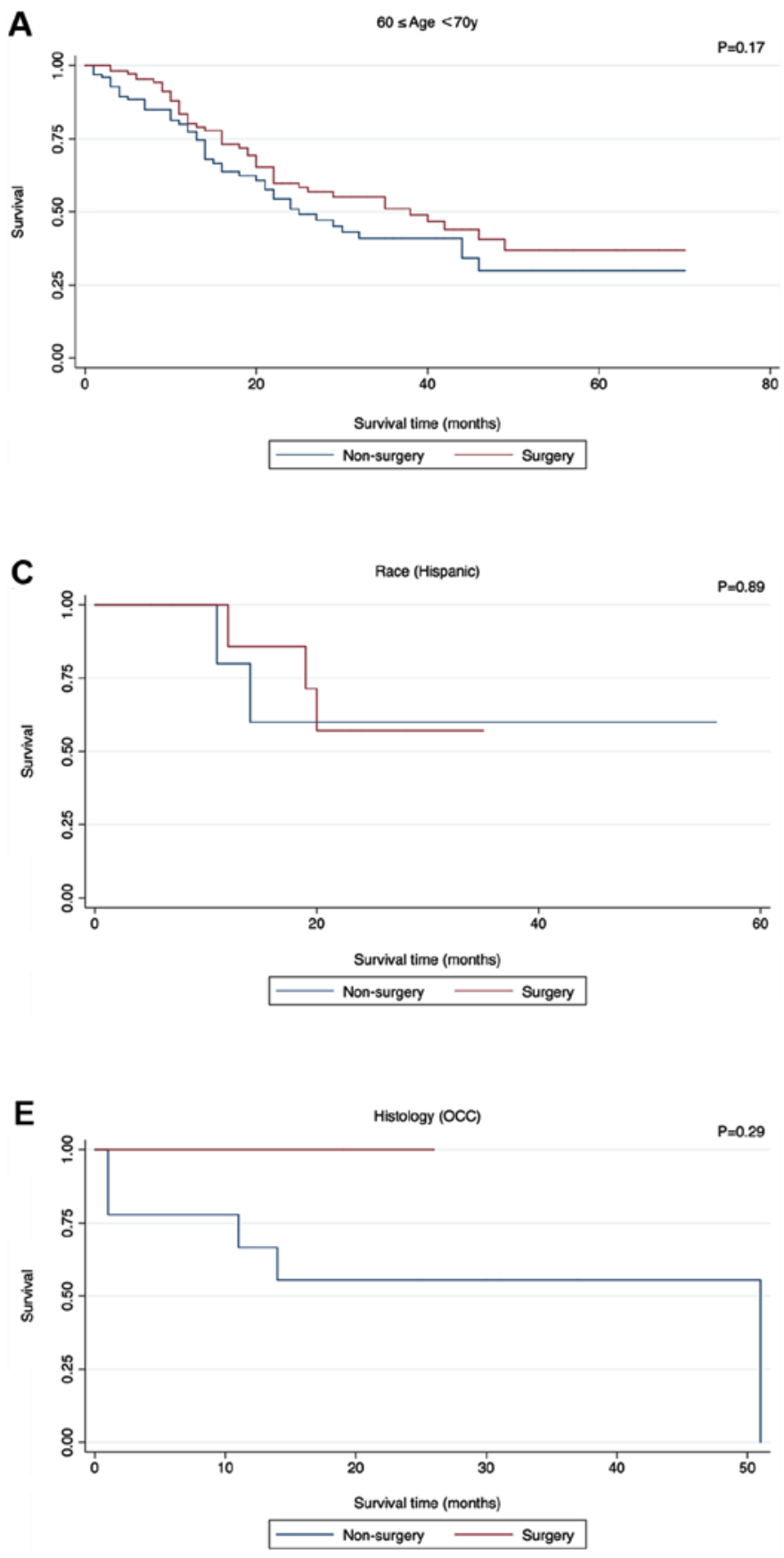
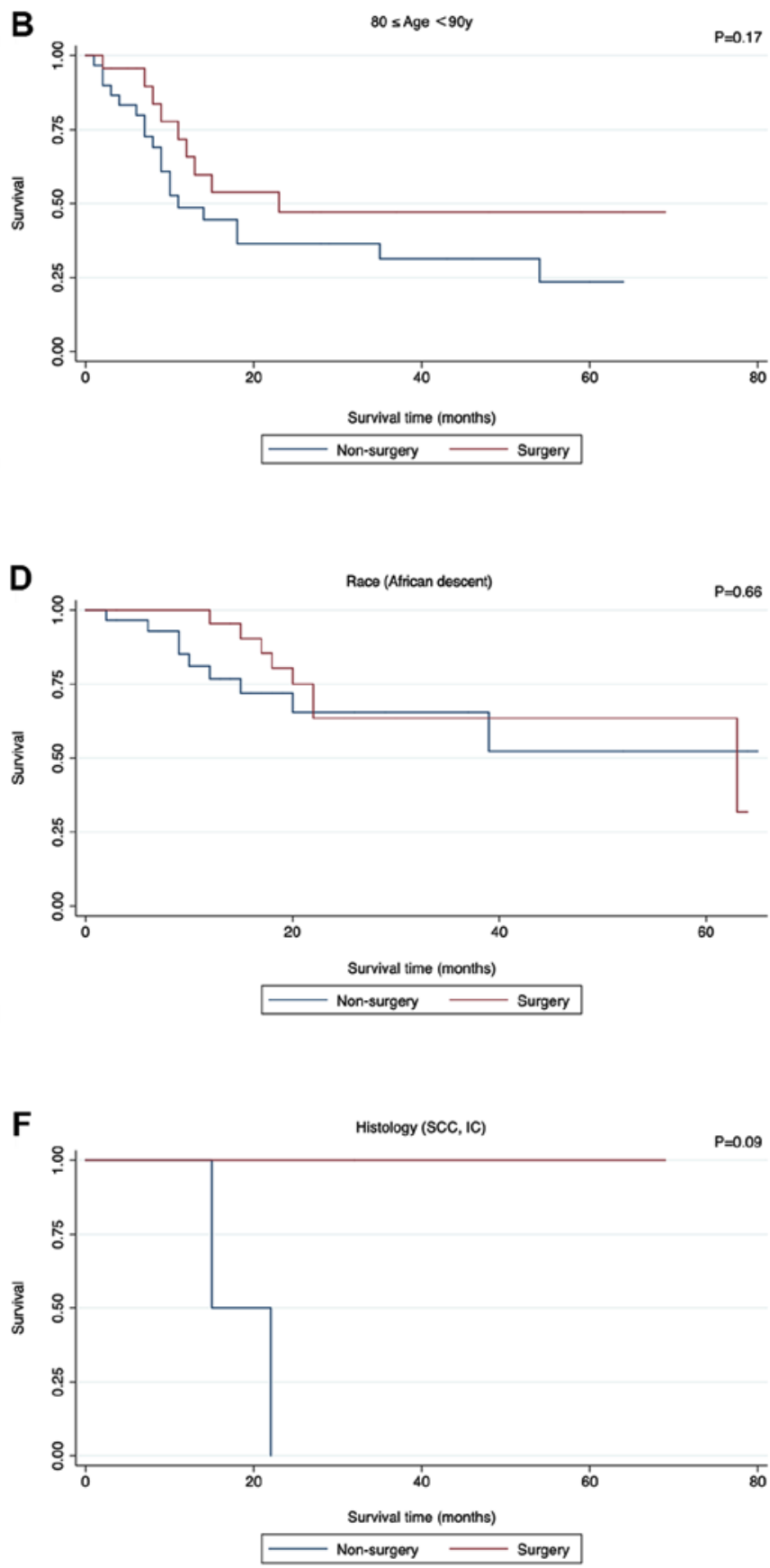

Figure 6. Insignificant survival functions of surgery stratified by clinicopathological features when comparing Non-surgery with surgery. (A) Age, $\geq 60$ and $<70$ years; (B) age, $\geq 80$ and <90 years; (C) race, Hispanic; (D); race, African descent; (E); histology, OCC; (F) histology, SCC, IC. OCC, Oat cell carcinoma; SCC, IC, Small cell carcinoma, Intermediate cell; CSCC, Combined small cell carcinoma.

there was no significant difference, it was the patients with SCLC with T1a to T2b, who had received lobe/s, who exhibited better survival trends. Simultaneously, the present study also demonstrated that more patients with N0-1, stage Ia-IIb who received sublobectomy, rather than lobe/s, were at risk of COD. Despite the recommendation in the most recent $\mathrm{NCCN}$ guidelines that patients with SCLC with clinical stage I-IIA (T1-2, N0, M0) after a standard staging evaluation may be considered for surgical resection (9) and multiple medical societies concluding that the survival advantage of surgical resection is only observed in patients with stage I disease $(5,10,18,19)$, the results of the present study suggest that patients with SCLC with up to stage IIB (N1) may benefit from lobe/s. Combs et al (20) also stated that patients with stages I, II and III SCLC that underwent surgical resection as part of the initial treatment with chemotherapy may exhibit an improved overall survival rate.

Due to the inclusion criterion, only 10 patients who received pneumonectomy were included in the present study, and therefore results were too limited to be extensively discussed. Despite the large sample size, a limitation of the SEER database, and consequently of the present study, was the lack of information regarding performance and smoking status, which may have an important impact on postoperative survival, and the use of perioperative effective treatments, 

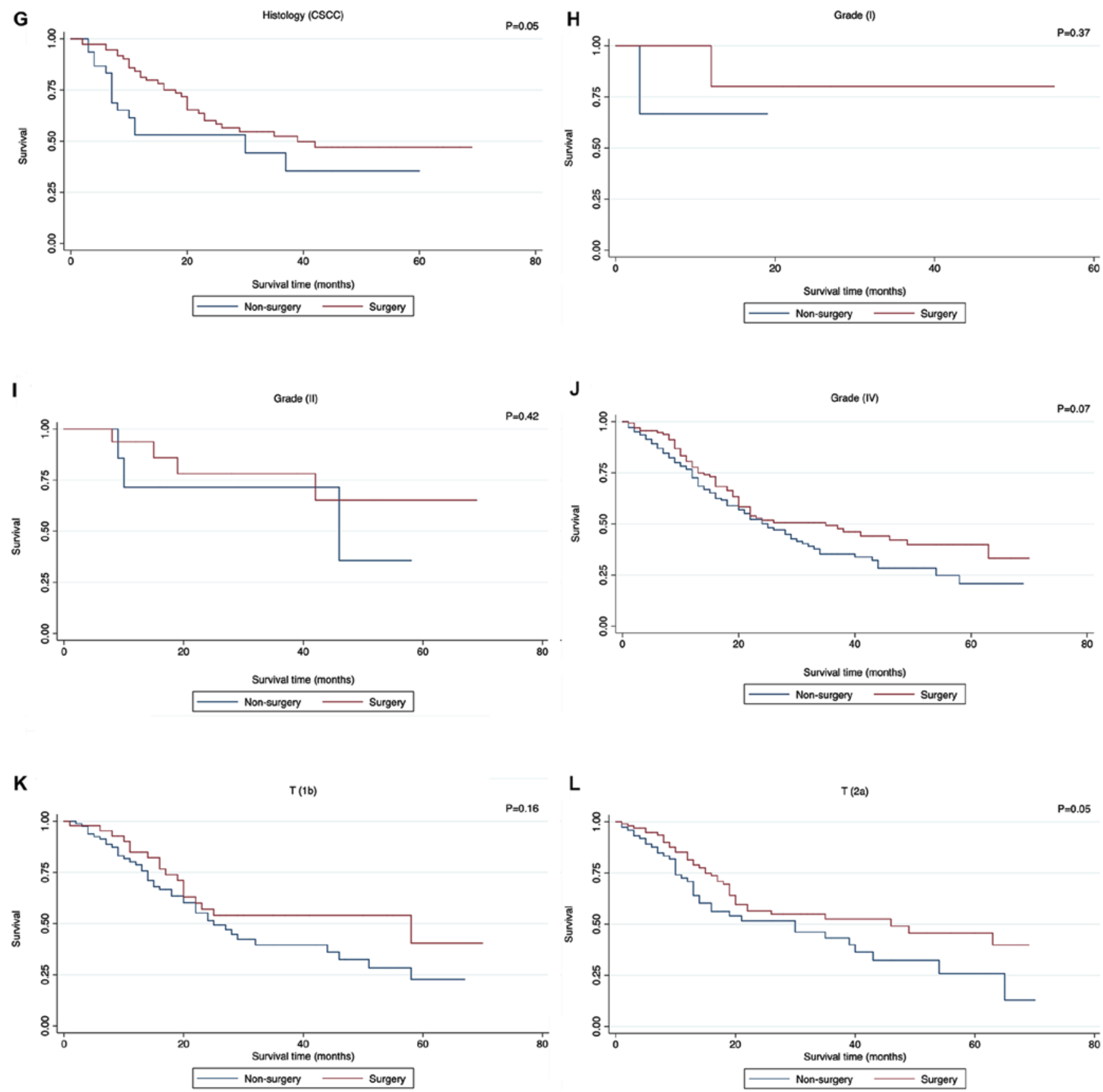

Figure 6. Continued. (G) Histology (CSCC); (H) Grade I; (I) Grade II; (J) Grade IV; (K) T1b; (L) T2a. CSCC, Combined small cell carcinoma.

including systemic therapy, mediastinal radiation therapy and prophylactic cranial irradiation. In addition, the different surgical types of lobectomy and bilobectomy were recorded as a single category 'lobectomy or bilobectomy', and the present study was unable to analyze the difference between them.

In conclusion, surgery should be taken into consideration when initial treatment strategy is made in patients with SCLC with a clinical stage I-IIA (T1-2, N0, M0), and should not be overlooked in patients $>50$ years, irrespective of sex, histology and the grade of the clinicopathological features. There is also evidence to suggest that certain patients with SCLC with stage IIB (N1) may also benefit from lobectomy or bilobectomy, although further investigation is required. In addition, lobe/s is preferred compared with sublobectomy when surgery is performed. However, the present study was unable to conclusively state the role of pneumonectomy for SCLC.

\section{Acknowledgements}

The authors would like to thank the Surveillance, Epidemiology, and End Results (SEER) Program for providing free access to the database.

\section{Funding}

This study was funded by the Wu Jieping Medical Foundation (grant no. 320.6750.18470) and the Qi Lu Cancer Research Foundation of Chinese Society of Clinical Oncology (grant no. Y-Q201801-006). 

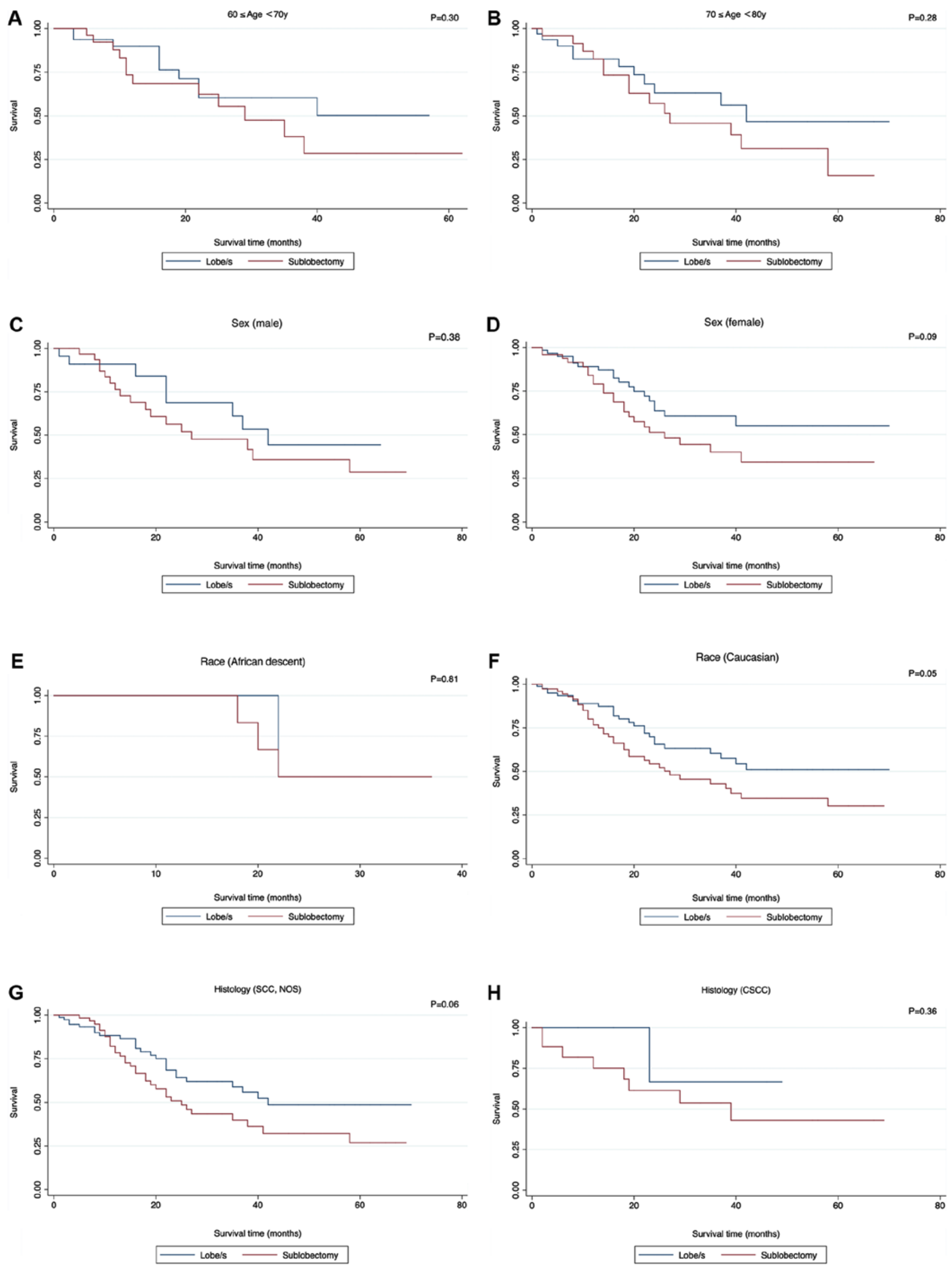

Figure 7. Insignificant survival functions of surgery stratified by clinicopathological features when comparing Sublobectomy with Lobe/s. (A) Age, $\geq 60$ and $<70$ years; (B) age, $\geq 70$ and <80 years; (C) sex, male; (D), sex, female; (E) race, African descent; (F) race, Caucasian; (G) histology, SCC, NOS; (H) histology, CSCC; SCC, small cell cancer; NOS, not otherwise specified; CSCC, combined small cell carcinoma. 
I
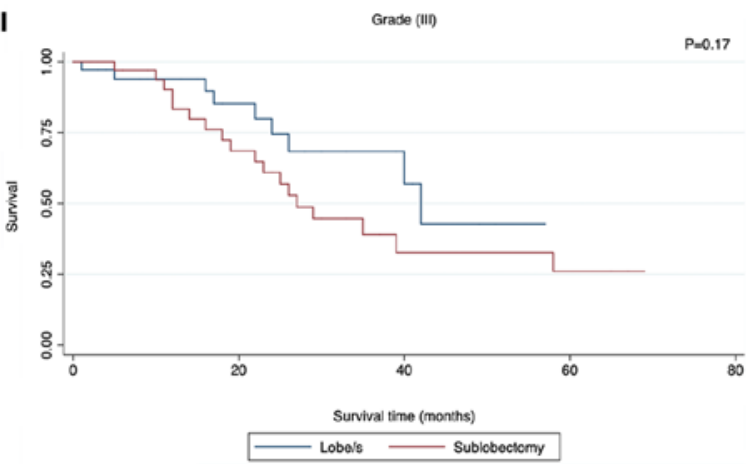

K

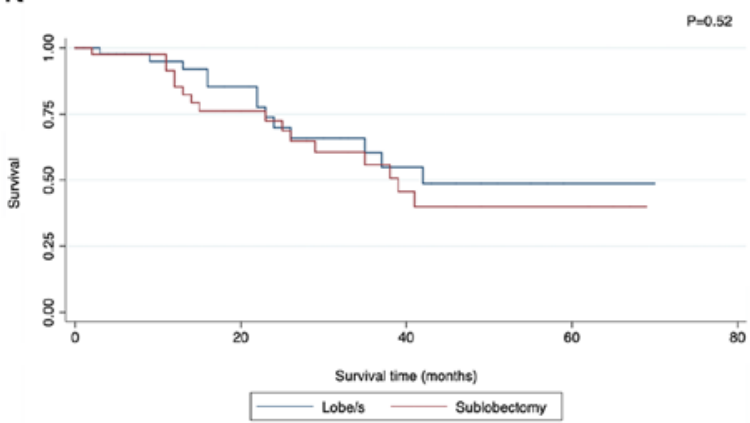

$J$
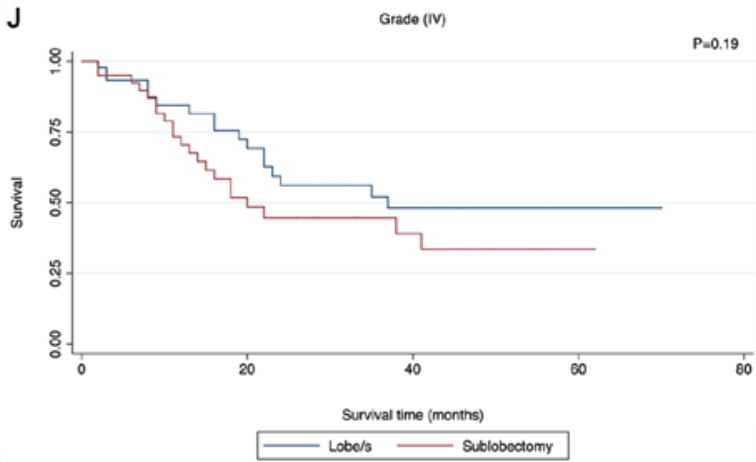

L

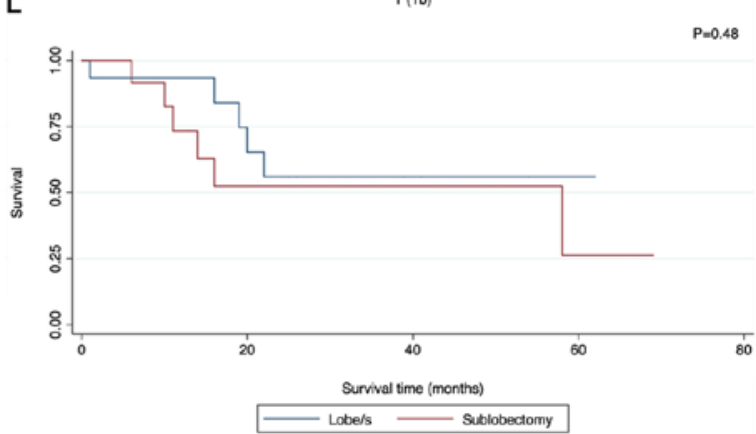

Figure 7. Continued. (I) grade III; (J) grade IV; (K) T1a; (L) T1b. SCC, small cell cancer; NOS, not otherwise specified; CSCC, combined small cell carcinoma.
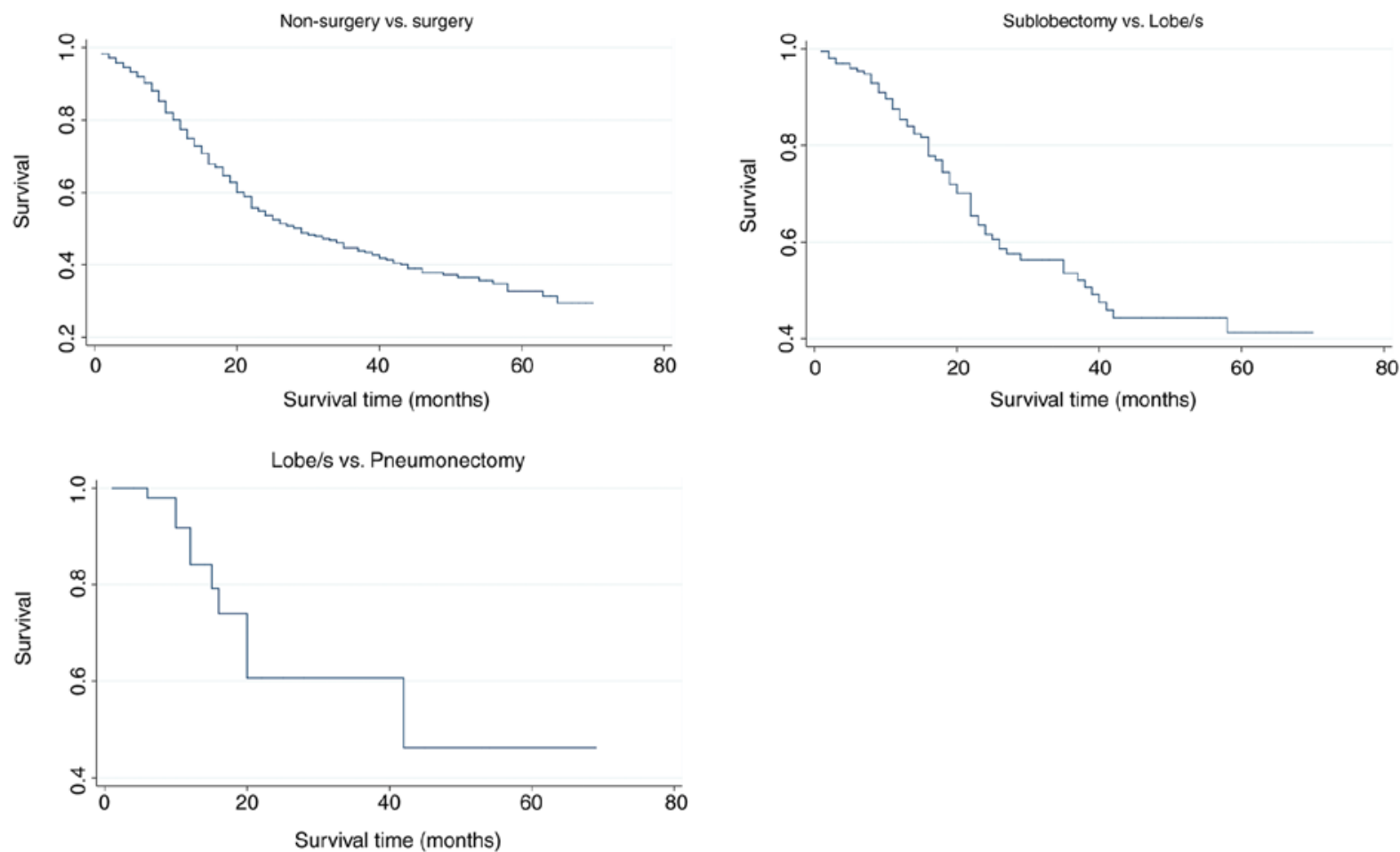

Figure 8 . The cumulative survival curves at the mean value of the covariates. Lobe/s, Lobectomy or bilobectomy.

\section{Availability of data and materials}

The datasets generated during and/or analyzed during the current study are available in the Surveillance, Epidemiology, and End Results (SEER; www.seer.cancer.gov) Program SEER*Stat Database.

\section{Authors' contributions}

XD, DT, JX, WL, SY, HZ and JZ participated in the design of the study and performed statistical analysis. LL, ZT and $\mathrm{XC}$ contributed to the acquisition and interpretation of data and critically revised the article for intellectual content. All 
authors were involved in the writing of the manuscript and approved the final version of the manuscript.

\section{Ethics approval and consent to participate}

Not applicable.

\section{Patient consent for publication}

Not applicable.

\section{Competing interests}

The authors declare that there is no competing interests.

\section{References}

1. Govindan R, Page N, Morgensztern D, Read W, Tierney R, Vlahiotis A, Spitznagel EL and Piccirillo J: Changing epidemiology of small-cell lung cancer in the United States over the last 30 years: Analysis of the surveillance, epidemiologic, and end results database. J Clin Oncol 24: 4539-4544, 2006.

2. Kalemkerian GP and Schneider BJ: Advances in small cell lung cancer. Hematol Oncol Clin North Am 31: 143-156, 2017.

3. Pleasance ED, Stephens PJ, O'Meara S, McBride DJ, Meynert A, Jones D, Lin ML, Beare D, Lau KW, Greenman C, et al: A small-cell lung cancer genome with complex signatures of tobacco exposure. Nature 463: 184-190, 2010.

4. Stinchcombe TE and Gore EM: Limited-stage small cell lung cancer: Current chemoradiotherapy treatment paradigms. Oncologist 15: 187-195, 2010

5. Takenaka $\mathrm{T}$, Takenoyama $\mathrm{M}$, Inamasu $\mathrm{E}$, Yoshida $\mathrm{T}$, Toyokawa G, Nosaki K, Hirai F, Yamaguchi M, Shimokawa M, Seto $\mathrm{T}$ and Ichinose Y: Role of surgical resection for patients with limited disease-small cell lung cancer. Lung Cancer 88: $52-56,2015$.

6. Schneider BJ, Saxena A and Downey RJ: Surgery for early-stage small cell lung cancer. J Natl Compr Canc Netw 9: 1132-1139, 2011.

7. Allen PW: ICDO-international classification of diseases for oncology. Pathology 23: 280, 1991.

8. Edge SB, Byrd DR, Compton CC, Fritz AG, Greene FL and Trotti A (eds). AJCC Cancer Staging Manual. 7th edition. Springer, New York, NY, 2010.

9. National Comprehensive Cancer Network. NCCN Clinical Practice Quidelines in Oncology (NCCN guidelines): Small Cell Lung Cancer, 2018. https://www.nccn.org/professionals/physician_gls/pdf/sclc.pdf. Accessed October 22, 2018.
10. Jett JR, Schild SE, Kesler KA and Kalemkerian GP: Treatment of small cell lung cancer: Diagnosis and management of lung cancer, III ed: American College of chest physicians evidence-based clinical practice guidelines. Chest 143 (Suppl 5): e400S-e419S, 2013.

11. Schreiber D, Rineer J, Weedon J, Vongtama D, Wortham A, Kim A, Han P, Choi K and Rotman M: Survival outcomes with the use of surgery in limited-stage small cell lung cancer: Should its role be re-evaluated? Cancer 116: 1350-1357, 2010.

12. Nicholson AG, Chansky K, Crowley J, Beyruti R, Kubota K, Turrisi A, Eberhardt WE, van Meerbeeck J and Rami-Porta R; Staging and Prognostic Factors Committee, Advisory Boards, and Participating Institutions; Staging and Prognostic Factors Committee Advisory Boards and Participating Institutions: The international association for the study of lung cancer lung cancer staging project: Proposals for the revision of the clinical and pathologic staging of small cell lung cancer in the forthcoming eighth edition of the TNM classification for lung cancer. J Thorac Oncol 11: 300-311, 2016.

13. Tas F, Ciftci R, Kilic L and Karabulut S: Age is a prognostic factor affecting survival in lung cancer patients. Oncol Lett 6: 1507-1513, 2013.

14. Allan SG, Stewart ME, Love S, Cornbleet MA, Smyth JF and Leonard RC: Prognosis at presentation of small cell carcinoma of the lung. Eur J Cancer 26: 703-705, 1990.

15. Lara JD, Brunson A, Riess JW, Kelly K, Lara PN Jr and Gandara DR: Clinical predictors of survival in young patients with small cell lung cancer: Results from the California cancer registry. Lung Cancer 112: 165-168, 2017.

16. Quon H, Shepherd FA, Payne DG, Coy P, Murray N, Feld R, Pater J, Sadura A and Zee B: The influence of age on the delivery, tolerance, and efficacy of thoracic irradiation in the combined modality treatment of limited stage small cell lung cancer. Int J Radiat Oncol Biol Phys 43: 39-45, 1999.

17. Paximadis P,Beebe-Dimmer JL, George J, Schwartz AG, Wozniak A and Gadgeel S: Comparing treatment strategies for stage I small-cell lung cancer. Clin Lung Cancer 19: e559-e565, 2018.

18. Rudin CM, Ismaila N, Hann CL, Malhotra N, Movsas B, Norris K, Pietanza MC, Ramalingam SS, Turrisi AT III and Giaccone G: Treatment of small-cell lung cancer: American society of clinical oncology endorsement of the American college of chest physicians guideline. J Clin Oncol 33: 4106-4111, 2015.

19. Inoue M, Sawabata N and Okumura M: Surgical intervention for small-cell lung cancer: What is the surgical role? Gen Thorac Cardiovasc Surg 60: 401-405, 2012.

20. Combs SE, Hancock JG, Boffa DJ, Decker RH, Detterbeck FC and Kim AW: Bolstering the case for lobectomy in stages I, II and IIIA small-cell lung cancer using the national cancer data base. J Thorac Oncol 10: 316-323, 2015.

(i) $(-)$ This work is licensed under a Creative Commons Attribution-NonCommercial-NoDerivatives 4.0 International (CC BY-NC-ND 4.0) License. 